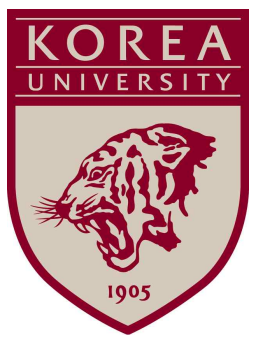

Discussion Paper Series

No. 1604

July, 2016

\title{
Organizational Design with Non-Contractible Quality
}

\author{
Seung Han Yoo
}

The Institute of Economic Research - Korea University

Anam-dong, Sungbuk-ku, Seoul, 136-701, South Korea, Tel: (82-2) 3290-1632, Fax: (82-2) 928-4948

Copyright (C) 2020 IER. 


\title{
Organizational Design with Non-Contractible Quality*
}

\author{
Seung Han Yoo ${ }^{\dagger}$
}

November 2022

\begin{abstract}
When contracting with an agent who is a worker of non-contractible quality, a principal considers mechanisms with an informed third party, a manager. To induce the manager with limited liability to report worker quality truthfully, the principal devises the first-order alignment, an incentive alignment based on the first-order condition with an interval structure. We show that the mechanism of contracting simultaneously with the manager and the agent dominates the optimal "selling the project" mechanism at a low information cost. The interplay between information cost and limited liability results in three optimal organizational structures: simultaneous contracting (manager inside the firm), ex ante contracting (out-sourcing), or partial contracting (no manager). Lastly, we apply this model to explain what may cause the difference in the firm structure across three types of labor market.
\end{abstract}

Keywords and Phrases: Non-contractible quality, first-order alignment, boundary of the firm, information cost, limited liability

JEL Classification Numbers: D21, D82, D86

${ }^{*}$ This is a substantially revised version of the paper "Mechanism Design with Non-Contractible Information" or "Mechanism Design with Two Types of Information." I thank participants at various conferences for comments on previous versions. This work was supported by a National Research Foundation of Korea Grant funded by the Korean government (NRF-2014S1A5A8018477). Of course, all remaining errors are mine.

${ }^{\dagger}$ Department of Economics, Korea University, 145 Anam-ro, Seongbuk-gu, Seoul, Republic of Korea, 02841 (e-mail: shyoo@korea.ac.kr). 


\section{Introduction}

Every real-world organization has an organizational structure, and one main role of the structure is to facilitate information acquisition and its transmission within it. This is especially important with regard to those aspects of work quality that are difficult to measure. For instance, a principal can contract on the number of reports or pages written by an agent, but it is hard to verify the quality, content, correctness, etc. Computer code may be another example; it is easy to contract on the number of lines written, but harder to verify their quality, robustness, adaptability, readability and reuse by other programmers. Needless to say, determining work quality - or type in general - is crucial to an organization's viability. To overcome such information asymmetry between firms and workers, employers typically consult a third party who has expertise to verify it. Job assignment, promotion, and other internal labor allocations are often constrained by subjective evaluations rather than measurable outcomes. ${ }^{1}$

The existing theories model worker quality in two ways: not observable and private value or observable investment but not verifiable. In a screening or signaling approach, worker quality is not a worker's private value, but the quality is paired with production cost - a worker's private value - so eliciting the cost through the single-crossing reveals the quality. In an incomplete contract approach, worker quality is a worker's human capital investment outcome, and this observable but nonverifiable action is settled through a bargaining process. Neither approach typically requires such a third party observing the quality for truthful revelation, however.

This paper suggests a model in which an agent's output consists of two tasks - quantity (e.g. number of pages or lines) and quality - such that the quality is observed privately by the agent but it directly affects the principal. With the model, we aim to provide a rationale behind an organizational structure with a third party. To illustrate the not-observable and not-private-value quality, consider an example:

A firm's owner assigns different hours to workers for a job. The job consists of two tasks, working hours (quantity), and value per hour (quality), since its unit output depends on each worker's quality.

The quality is not contractible because it is the agent's private information, and it is not possible to elicit it either because the agent has a multi-dimensional type consisting of quality and production cost of quantity, based on the multi-task. The quality is the agent's private information whereas the quantity is observable, since the former is difficult to measure, unlike the observable measures for the latter. ${ }^{2}$ Such quality, in the classical multi-tasking model of Holmström and Milgrom (1991), can be found not as an agent's type but as an outcome of an agent's action that is difficult to

\footnotetext{
${ }^{1}$ Anecdotal evidence well documented by Baker, Gibbons and Murphy (1994) not only supports the case that performance is not easily measurable, as intended in their paper, but also the case that worker quality cannot be assessed.

${ }^{2}$ If the relationship between two parties is one-time, as in a standard adverse selection model such as the buyerseller setting where a seller's product quality, e.g., a used car's quality, also directly affects a buyer, the quality is not contractible with a different rationale; typical terms of trade protect a seller so that a buyer cannot make payment contingent on it, that is, paying after using a product. To resolve such non-contractibility, reputation building, as in Kreps (1990), can be used if an uninformed party can observe an outcome after each period, but in this model, a quality outcome is difficult to measure, at least in the short term.
} 
measure. The principal of the present paper cannot screen the quality at all via the revelation principle (e.g., Myerson (1982)), without a third party, since it is not the agent's value but the "principal's value" in the sense that it affects not the agent's payoff but the principal's - so it does not appear in the former's payoff function. Then, the principal can only offer a contract that is constant over quality, which we call "partial contracting." This means, in the example, that despite potential differences in worker quality, each worker is assigned to the job for the same number of hours, according to a system of piece rates. The quality's direct effect on the principal's payoff, one should recall, is a standard feature in typical screening or signaling models, but the single task formulation with the aforementioned pairing in them simply makes quality revealed through cost revelation.

The principal, thus, cannot make any contract for the agent (or any third party) contingent on the (true) worker quality, but he can make it contingent on a report on quality. That is, the contractual incompleteness drives the principal to consider a manager to acquire the information. ${ }^{3}$ Unlike the agent, however, the manager incurs costs when seeking to acquire the information after participating in the organization, as the quality is not his intrinsic type, and he is protected by limited liability. Such role of a third party observing an agent's type was originated by Tirole (1986). ${ }^{4}$ The quality as a separate task in this paper departs from the seminal paper in which an agent's action and type are accrued to a one-dimensional task. In other words, the role of a third party in the present paper is to report non-contractible quality, which is related to subjective evaluations (see, Levin (2003), MacLeod (2003), and Fuchs (2007)). The key difference between this model and relational contracts is a type of information structure. In this paper, the quantity is observable and verifiable, and with the manager, the quality is contractible, which enables us to use a mechanism design approach. That is, the approach with relational contracts studies relevant environments in which outcomes are nonverifiable, whereas this paper studies environments akin to the information structure by Holmström and Milgrom (1991) such that one aspect is contractible, but the other aspect is not. ${ }^{5}$ The difference is not just to make it depart from the said approach but most importantly to make the present model serve for the main purpose of this paper - the optimality of including a third party inside an organization by the interplay between information (acquisition) cost and limited liability. That is, the principal aims to minimize the manager's compensation, which results in the trade-off between information acquisition and an extra rent to the manager since the rent is a transfer between them. ${ }^{6}$ In addition, the nature of monitoring in this paper is to screen the agent's information, not moral hazard.

The benchmark of this framework is the second-best case - maximum possible payoff - in which

\footnotetext{
${ }^{3}$ The principal could also have expertise to verify worker quality, but he may not have time to do so. See an expository writing, Arrow (1964), for the relevance of the problem, called "the span of control."

${ }^{4}$ In addition, the manager's payoff is only monetary compensation from the principal, as in Tirole (1986).

${ }^{5}$ We define the non-measurable quality as the type counterpart of the non-measurable action from Holmström and Milgrom (1991) in the sense that different parties can agree on the same quality ranking, but the problem is that true quality one possesses is hard to measure. By modifying their leading example, in the present paper, enhancing students' creative thinking is not about an action taken by a teacher but about how qualified the teacher is for such a task. The teacher's skills cannot be measured because the direct effects of teacher quality on students' development of higher-order thinking are only realized in the long term, unlike observable teaching hours or test scores.

${ }^{6}$ On the other hand, the total payment must be fixed via the standard "money burning" in relational contracts.
} 
the principal, like the manager, can acquire information on worker quality, while production cost remains the agent's private information. Without information cost, achieving the second best is almost trivial; a constant payment induces the manager to report the quality truthfully, leaving no surplus to him, but even an infinitesimally small positive cost discourages the manager from acquiring information on it. The same problem arises for other mechanisms making use of the fact that the quality is known both to the agent and the manager, for example, punishing them for two different quality reports. ${ }^{7}$ Another convenient solution is the selling-the-project mechanism, called ex ante contracting. However, if the manager is protected by (ex post) limited liability, the ex ante contracting mechanism can be suboptimal. ${ }^{8}$

This paper provides three main results: characterization of the optimal mechanism, endogenous choice of an interval structure for the mechanism, and their implications for the boundary of a firm. We start by observing that the well-known simple contracts fail to incentivize the manager's information acquisition because in this model, the principal must tackle combinatorial deviations: The manager can misreport the agent's quality after not acquiring the information. ${ }^{9}$ That is, for the optimal contract to achieve the second best, the principal must provide truthful incentive compatibility together with an incentive for information acquisition to resolve the problem. On the other hand, to circumvent the direct effect of limited liability of selling the project, any base compensation for limited liability has to be decoupled from incentive compatibility. Thus, providing truthful incentive compatibility in this model is a delicate problem due to the incentive for information acquisition and limited liability. It is not just for its theoretical contribution per se but, more interestingly, for its implications for the boundary of a firm.

We propose a mechanism of contracting simultaneously with both the manager and the agent to handle combinatorial deviations and the decoupling. For the manager's monetary compensation - in the absence of manager's innate payoff function - the principal aligns the manager's optimality condition for truth-telling with the principal's optimality for the second best, through the first-order condition. We term it the first-order alignment. The incentive alignment is in fact shown to be achieved with a natural contractual form. The principal sets a target quantity, and if the agent's quantity turns out to be greater than that, he rewards the manager such that the compensation depends on the manager's report on quality. Thus, it has an interval structure for observable volume of output, generalizing the interval delegation by Holmström $(1977,1984)$. The necessary condition of reporting worker quality truthfully becomes sufficient by connecting it to the reverse hazard rate dominance between the two dimensions of a type, quality and cost, without an exogenously given

\footnotetext{
${ }^{7}$ Another potential problem with the scheme is collusion through communication between them.

${ }^{8}$ Selling and a selling-like contract cannot explain a firm's structure based on the informational aspect because it fails to show the emergence of an integrated large firm in reality, instead of multiple disintegrated firms. The ex ante contract can be interpreted in two ways; a contract within an organization is executed in exactly the same way as selling the project, and the project is "literally" sold. In the former, the principal retains ownership; in the latter, ownership of the project is transferred to the manager. Nonetheless, retaining the ownership combined with the selling does not require any information flow between them, so the two are treated virtually the same. The limited liability in the former can be translated into outsourcing with limited liability or selling with liquidity constraint in the context of the latter.

${ }^{9}$ See Myerson (1982) for such deviations and the difference between the truthful or honest incentive compatibility and the obedience incentive compatibility.
} 
single-crossing. ${ }^{10}$ The first main result shows that if information acquisition is not very costly, the simultaneous contracting mechanism attains the second-best payoff, dominating the selling. ${ }^{11}$

Next, we turn to the question of finding an optimal interval structure (or target quantity levels), which determines the maximum information cost that admits a simultaneous contracting mechanism. This requires solving a minimax problem in which the principal minimizes the manager's maximum payoff when he does not acquire the information, deviation payoff. ${ }^{12}$ Then, if the information cost is greater than the maximum admissible cost from the minimax problem, the simultaneous contracting is not feasible.

The characterization of the maximum admissible cost enables us to identify conditions under which the simultaneous contracting or the ex-ante contracting dominates. The limited liability affects the price for selling-the-project - the principal's payoff from the ex-ante contracting - directly, whereas the first-order alignment eliminates such a constraint on the principal's payoff. Yet, the alignment at the same time is linked to the deviation payoff, which results in a bound for the admissible cost. However, the bound can be loosened with an extra rent to the manager, which leads the principal to face the trade-off between information acquisition and the rent. Thus, the key insights for either contract's dominance rely on the interplay between the extent to which the limited liability constrains the principal and the magnitude of information cost.

The trade-off provides a rich outcome on the boundary of the firm such that, depending on the cost and the size of limited liability, each of the three mechanisms - simultaneous, ex ante and partial contracting - can be optimal. The three mechanisms emerge as three corresponding organizational structures: (1) if the information cost is below a threshold that depends on the extent of limited liability, the optimal structure is to include the manager inside a firm; (2) if it is intermediate, then outsourcing is best, and (3) if it is high, no quality assessment is optimal. The ex-ante contracting may not be a profitable option at all if the negative effects from limited liability are severe. The analysis can be further extended to answer an intriguing question raised by Holmström and Roberts (1999) about the difference in the firm structure between U.S. firms (exercising simultaneous contracting more) and Japanese firms (exercising ex-ante contracting more), in the presence of the hold-up problem.

We discuss the related literature in the following section. Section 3 introduces a model, and Section 4 reports preliminary results. The dominance of the simultaneous contracting is provided in Section 5 and the optimal interval structure in the following section. The boundary of the firm is analyzed in Section 7. Section 8 concludes, and the proofs are collected in an appendix.

\footnotetext{
${ }^{10}$ The cost distribution conditional on a higher quality dominates the one on a lower quality with respect to the reverse hazard rate, which is widely used in auction theory (see, Maskin and Riley (2000) and Kirkegaard (2012)) for ranking auctions, but no paper applied it to eliciting a type.

${ }^{11}$ At least two more reasons for the dominance of a simultaneous contracting mechanism over selling can be suggested: management cost and partial commitment. First, if the principal is an expert in management, only the manager incurs a "management cost" when he becomes the mechanism designer (buys the project). Second, within an organization, people lower in the hierarchy (like the manager relative to the principal) typically have more specific information but less commitment power.

${ }^{12}$ Further, under a reasonable condition - differentiability combined with single-peak property of two intervals we can show that the optimal number of intervals is simply two, as discussed in Appendix B.3.
} 


\section{Related Literature}

Study on a third party inside an organization has been a subject of interest over the last several decades. The third party's role is to monitor an agent (or agents) in the organization owned by a principal, and the role can be further classified into two cases: monitoring the agent's action or type. Alchian and Demsetz (1972) propose a monitor observing agents' actions in a team, suggesting monitoring as an origin of a firm's structure. Tirole (1986) introduces a supervisor observing an agent's type in the three-tier hierarchical structure, with its focus on collusion between them; monitoring is essential as there is no collusion with selling. ${ }^{13}$ Unlike both papers, the principal's goal in the present paper is to solve a screening problem: how the principal can assign different volumes of output to a worker of varying quality with the manager's report, not how to incentivize a worker to exert certain efforts. ${ }^{14}$ Furthermore, a principal in the present paper hires a manager observing an agent's quality, as in the latter, but with a different model and purpose. In Tirole (1986), the agent's type and action are added to produce a one-dimensional output, and monitoring incurs no cost. In the present paper, the agent's action and type represent two distinct dimensions of a single output, quantity and quality, in addition to two-dimensional private information, and equally importantly, a manager incurs an information cost for monitoring, while protected by limited liability.

The present paper's main purpose is to provide the rationale for why a third party is inside an organization in the first place: the optimality of the third party inside the firm. Despite the resonating influence of such a firm structure from its inception, the question has been rather neglected in the literature. We answer the question with non-contractible quality and two key factors: information cost and limited liability. The interplay between them yields three optimal organizational structures, while a single factor fails to account for different structures; without limited liability, the optimal contract is always selling, and without information cost, the optimal contract must be to include a third party inside the firm, by having him acquire the information. Such a "mix" of costly information acquisition and limited liability, naturally adopted in this paper to provide a firm's origin, has not been investigated, so it is a separate theoretical contribution. Still, the present paper treats information cost and limited liability differently from previous papers in each of the environments.

The different treatment of the two well-known environments is due to non-contractibility of quality in the information structure of Holmström and Milgrom (1991). In the multitasking model by Holmström and Milgrom (1991), if one activity cannot be measured at all, incentivizing the other activity that is measured can affect the effort made for the unobservable action through their interactions from a cost function (for example, two activities can be complements or substitutes). In this model, one dimension, worker quality, is the agent's type, not an action. Thus, an interaction between two dimensions to induce the manager's truthful report is provided through correlation

\footnotetext{
${ }^{13}$ There is a vast literature on collusion with a third party following Tirole (1986); we wish to relate them to the future research of this model as commented in the concluding remarks.

${ }^{14}$ That is, unlike the standard moral hazard models, there is no observable noisy signal from an action in this screening model, which differs from models with such a signal structure and extended models with mediated contracts, e.g. in Rahman (2012) and Strausz (2012) among others.
} 
between the observable volume of output and worker quality, not through a cost function. ${ }^{15}$ An interesting, recent development of Bénabou and Tirole (2016) includes screening into the multitasking model by incorporating a talent parameter or an intrinsic motivation as a type of an agent. Since the type is associated with a contractible action in their paper, a screening approach can be adopted for truthful revelation of the type, but in this model, without a third party observing worker quality, the principal cannot screen it at all. In a seller and a buyer setting, Schmitz (2002) establishes a powerful result, related to this finding, in which the first best may not be achieved due to two types of post-contractual informational asymmetries arising from the combination of hidden action and hidden information, but both of this model's two dimensions are hidden information.

The costly information acquisition in a production model of Baron and Myerson (1982) was first introduced by Crémer, Khalil and Rochet (1998a,b). This paper also considers a Baron-Myerson setting as the baseline model, but unlike their papers, in the present paper, it is a third-party, the manager, who acquires the agent's information in a three-tier hierarchical model as in Tirole (1986). Although this paper shares some common features with them such as the production model and the optimal mechanism to induce information acquisition, we focus on the third-party's information acquisition. ${ }^{16}$ Ben-Porath, Dekel and Lipman (2014) suggest a different approach on mechanisms with costly information acquisition such that the principal himself can incur a cost to verify each agent's private information. Like the present paper, although the agent's information affects a principal's payoff, the information remains the agent's private information. The present paper differs from the approach since the principal of this model cannot check the quality due to either lack of expertise or lack of time to do so or both (see the span of control in Arrow (1964)), and secondly, they consider environments where there are no monetary transfers with relevant applications, whereas the present paper considers situations where it is possible to investigate different firm structures with a two-dimensional type and two different tasks - quantity and quality - for the agent.

In the presence of limited liability, Innes (1990) shows that the optimal contracts have a binary form with a threshold to motivate debt-style contracts, which is further generalized by Poblete and Spulber (2012), in particular, without the monotone likelihood ratio property. ${ }^{17}$ The optimal contracts with a threshold or an interval in that literature is to incentivize an agent's efforts, whereas

\footnotetext{
${ }^{15}$ Utilizing the conditional expectations with correlation between quality and cost is essential, as in Crémer and McLean (1988), but their mechanism, despite the generality, is not applicable to the manager because it does not consider limited liability; without limited liability, there is no reason to resort to them is found, with the convenient selling option in this model. In addition, the principal of the present paper has to tackle limited liability while incentivizing the manager's information acquisition.

${ }^{16}$ See, for example, Bergemann and Välimäki (2002) for the efficient mechanism in an auction model, where a Vickrey-Clarke-Groves (VCG) mechanism provides incentive compatibility for an agent - corresponding to the manager in the present paper - and its corresponding payoff incentivizes information acquisition, but the VCG mechanism for the manager of this model is a constant payment, resulting in a conflict between incentive compatibility and information acquisition. See, also, e.g., Caplin and Dean (2015) to grasp this assumption's latest foundation by a behavioral approach.

${ }^{17}$ The present paper's first-order alignment is conceptually different from the first-order approach for this literature as well as that on subjective evaluations: The alignment is to connect the manager's incentive with the principal's to induce truth-telling via their first-order optimality to tackle limited liability and further it provides the sufficiency for global optimality through the reverse hazard rate dominance.
} 
an interval structure from target quantity levels in the present paper is adopted to induce a third party's truth-telling for the agent's type. Thus, the nature of the problem that the present paper studies is different, so given that the manager is not involved in any production, we can obtain the optimal contracts as long as the manager's individual rationality is binding. Yet, the principal must incentivize the manager to acquire the information with a positive cost, together with inducing his truth-telling. Hence, choosing an optimal interval structure becomes a delicate problem when the cost is high; any structure with the binding individual rationality suffices otherwise.

This paper is also related to three more strands of literature. The quality is not observable by the principal, so a bargaining procedure in incomplete contract literature from the property rights approach by Grossman and Hart (1986) and Hart and Moore (1990) built on transaction costs (Williamson $(1975,1985)$ ) cannot be extended to this not-observable and not-private quality case. The manager's report on non-contractible quality is related to a relatively large literature on subjective evaluations, another form of non-contractible outcomes, following Levin (2003), MacLeod (2003), and Fuchs (2007). Deb, Li and Mukherjee (2016) further advance the approach to consider both nonverifiable public and private performance measures for moral hazard with a third party inside the firm, providing new insights into why the first-best outcome cannot be achieved if the third party and a worker both produce and monitor each other in a repeated game. The present paper proposes a third party for screening of quality, not moral hazard, and more importantly, we locate this model in the realm of mechanism design to focus on the optimality of a third party inside the firm with the role of different residual rewards to the manager - such a role also plays a key role in Alchian and Demsetz (1972). That is, with the manager, we resolve non-contractibility since the quantity is observable and verifiable, and as such, the driving force to shape a firm's structure in this model is the two key factors: limited liability and information cost. Last, monetary transfers make this model attain the delegation as an implementation of the mechanism with no friction, unlike the optimal delegation literature based on a bias as in Crawford and Sobel (1982). ${ }^{18}$

\section{Model}

A principal contracts with a manager and an agent (a worker). The agent produces $q \geq 0$ units of output with $\omega$ quality per unit of the product, so the agent's output consists of two dimensions: quality and quantity, $(\omega, q)$. The agent incurs cost $\theta q$ to produce $q$ quantity at marginal cost $\theta$, whereas quality $\omega$ is his endowed characteristic like $\theta$. A two-dimensional type $(\theta, \omega)$ is drawn from a non-empty subset $\Theta \times \Omega$ of $\mathbb{R}^{2}$, where $\Theta \equiv[\underline{\theta}, \bar{\theta}]$ and $\Omega \equiv[\underline{\omega}, \bar{\omega}]$. The principal obtains a value $v(\omega, q)$ that consists of observable (and verifiable) quantity $q$ and non-observable quality $\omega$. The quality $\omega$ is the agent's private information but affects the principal's payoff - principal's value. The principal cannot observe the quality since it is difficult to measure. ${ }^{19}$ The manager has the expertise to acquire information on the agent's quality $\omega$ at cost $c>0$.

If the principal assigns the agent of quality $\omega$ the production $q$ by making a monetary transfer

\footnotetext{
${ }^{18}$ The literature is relatively large to review them all in this paper.

${ }^{19}$ If the relationship is one time as in a seller-buyer setting, private information is sufficient. Thus, non-measurability provides a rationale for why it remains as the agent's private information even for other cases, including this model.
} 
$t$ to him, the agent obtains

$$
t-\theta q
$$

The quality $\omega$ does not appear above since although both marginal cost $\theta$ and quality $\omega$ are the agent's private information, but they differ in that, unlike the $\operatorname{cost} \theta$, the quality $\omega$ affects not the agent's payoff but the principal's. If the principal makes such assignment and transfer, he obtains

$$
v(\omega, q)-t
$$

where $v$ is twice-differentiable, strictly increasing in each of $\omega$ and $q$, strictly concave in $q$, and $\omega$ and $q$ are complementary. ${ }^{20}$ The total surplus of the principal and the worker is $v(\omega, q)-\theta q$, and for each $\omega$, we assume that $v_{q}(\omega, 0)>\underline{\theta}$ and $v_{q}(\omega, 0) \leq \bar{\theta}$ so that the former ensures that the principal has an incentive to have the worker produce if the cost is the lowest, and the latter ensures that he has no such incentive if it is the highest; and that $\lim _{q \rightarrow \infty} v_{q}(\omega, q)<\underline{\theta}$ so that the optimal output for any type of worker is finite.

The conditional cumulative distribution function of cost $\theta$ given quality $\omega$ is denoted by $F(\theta \mid \omega)$ with density function $f(\theta \mid \omega)>0$ for all $\theta \in \Theta$. We assume the standard monotone hazard rate condition for $F(\theta \mid \omega)$ for each fixed $\omega$; the ratio $\frac{F(\theta \mid \omega)}{f(\theta \mid \omega)}$ is nondecreasing in $\theta$. In addition, quality $\omega$ and $\operatorname{cost} \theta$ are related in such a way that for any pair $\omega^{\prime}>\omega, F\left(\theta \mid \omega^{\prime}\right)$ dominates $F(\theta \mid \omega)$ in terms of the reverse hazard rate, that is, $\frac{f\left(\theta \mid \omega^{\prime}\right)}{F\left(\theta \mid \omega^{\prime}\right)}>\frac{f(\theta \mid \omega)}{F(\theta \mid \omega)}$ for all $\theta \in(\underline{\theta}, \bar{\theta})$, which implies the first-order stochastic dominance. ${ }^{21}$ Each $\omega$ is drawn from a cumulative distribution function $G(\omega)$ with density function $g(\omega)>0$ for all $\omega \in \Omega$.

We denote by $\bar{U}$ the manager's reservation payoff, and by $B$ his ex post limited liability (or liquidity constraint), assuming that they are any two real numbers such that the reservation payoff is greater than the limited liability, $\bar{U}>B .{ }^{22}$ The agent's reservation payoff, on the other hand, is normalized to 0 . Finally, the agent observes quality $\omega$ without information cost before participating in a mechanism, but the manager must incur a cost to acquire information on it after participating. Hence, we consider an ex ante individual rationality for the manager. ${ }^{23}$

\section{Benchmark}

In this section, we provide two preliminary results. First, we suppose that there is no manager, and second, suppose that there is no manager but the principal can observe quality $\omega$. In particular, the latter's payoff will be a benchmark for the main analysis. Even if quality $\omega$ is observable, cost

\footnotetext{
${ }^{20}$ Its partial derivatives are $v_{\omega}>0, v_{q}>0$; the second partial derivative is $v_{q q}<0$; and the cross partial is $v_{\omega q} \geq 0$ for all $(\omega, q) \in \Omega \times \mathbb{R}_{+}$. Throughout the paper, we use a subscript to denote a partial derivative of a function.

${ }^{21}$ See Krishna (2002) for its property.

${ }^{22} \mathrm{~A}$ negative value for $B$ can have a more natural interpretation in the context: the manager is liable for a loss up to, say, $B=-\$ 100$ or constrained by liquidity up to $B$.

${ }^{23}$ As the owner of the organizational structure, the principal has a legitimate right to choose when to hire the manager and, by doing so, grant him the opportunity to acquire information on the agent's quality: Timing for the manager is chosen by the principal, not by "Nature," and it is clear that for the principal, hiring the manager in the ex ante stage weakly dominates hiring the manager in the interim.
} 
$\theta$ still remains the agent's private information, so the benchmark is called the second-best optimal payoff that the principal obtains, not the first best. ${ }^{24}$

\subsection{Partial contracting}

The principal, with no third party, designs a mechanism only with the agent. A two-dimensional type $(\theta, \omega)$ being the agent's private information, a direct mechanism $\Gamma^{p}$ consists of two-dimensional functions, $q$ and $t$, where $q: \Theta \times \Omega \rightarrow \mathbb{R}_{+}$and $t: \Theta \times \Omega \rightarrow \mathbb{R}$. In light of the revelation principle, the agent is asked to report $(\theta, \omega)$, and the principal assigns the agent the production of $q(\theta, \omega)$ and commits to paying him $t(\theta, \omega)$. A direct mechanism can be implemented such that the principal suggests a set of pairs $(q, t)$, leaving the agent to choose one, as a standard screening problem. A mechanism is said to be incentive compatible if for each $(\theta, \omega),\left(\theta^{\prime}, \omega^{\prime}\right) \in \Theta \times \Omega$,

$$
t(\theta, \omega)-q(\theta, \omega) \theta \geq t\left(\theta^{\prime}, \omega^{\prime}\right)-q\left(\theta^{\prime}, \omega^{\prime}\right) \theta .
$$

We show that, without the manager, it is impossible for the principal to assign different quantity allocations depending on different quality reports.

Proposition 1 For any mechanism between the principal and the agent, the incentive compatibility (3) requires that for each pair $\omega \neq \omega^{\prime} \in \Omega, q(\theta, \omega)=q\left(\theta, \omega^{\prime}\right)$ for almost all $\theta \in \Theta$.

The idea behind the result is straightforward. The quality does not appear in the agent's payoff, as mentioned in the model, so, to satisfy the incentive compatibility (3), the agent must obtain the same payoff for any two different quality reports, implying that different quality levels produce "essentially" an identical volume of output (quantity) for the same cost type. On the other hand, it is not trivial either as the result holds with any level of correlation between quality and cost.

With a constant output over quality, a partial contracting mechanism $\Gamma^{p}$ consists of $q_{p}(\theta)=$ $q(\theta, \omega)$ and $t_{p}(\theta)=t(\theta, \omega)$ for all $\omega$. The principal obtains expected payoff from $\Gamma^{p}$ such that

$$
V\left(\Gamma^{p}\right)=\int_{\Omega} \int_{\Theta}\left[v\left(\omega, q_{p}(\theta)\right)-q_{p}(\theta) \theta-\int_{\theta}^{\bar{\theta}} q_{p}(x) d x\right] f(\theta \mid \omega) d \theta d G(\omega),
$$

and a partial contracting mechanism $\Gamma^{p}$ is optimal if it maximizes the above payoff subject to incentive compatibility and individual rationality conditions for $\left(q_{p}(\theta), t_{p}(\theta)\right)$, which is denoted by $V_{p}$ (see the proof of Proposition 2 for a detailed procedure).

\subsection{Second best}

Now, suppose that the principal can acquire the agent's quality $\omega$ if he incurs the same opportunity cost and information (acquisition) cost, $\bar{U}+c$, like the manager. Then, a direct mechanism $\Gamma^{s}$ consists of functions, $q_{s}$ and $t_{s}$, where $q_{s}(\cdot, \omega): \Theta \rightarrow \mathbb{R}_{+}$and $t_{s}(\cdot, \omega): \Theta \rightarrow \mathbb{R}$. With the principal's information acquisition of quality $\omega$, the previous two-dimensional functions $q$ and $t$ become singlevariable functions, and for each observed $\omega$, the incentive compatibility condition given different values of $\theta$ becomes a "reverse" problem of the well-known nonlinear pricing (see, e.g., Mussa and

\footnotetext{
${ }^{24}$ Because the agent observes both dimensions, throughout our analysis, clearly, no rent extraction-type mechanism utilizing conditional probabilities is applicable to the agent.
} 
Rosen (1978)). A mechanism is said to be incentive compatible and individually rational if for each $\omega \in \Omega$, and every $\theta, \theta^{\prime} \in \Theta$,

$$
\begin{aligned}
& t_{s}(\theta, \omega)-q_{s}(\theta, \omega) \theta \geq t_{s}\left(\theta^{\prime}, \omega\right)-q_{s}\left(\theta^{\prime}, \omega\right) \theta, \\
& t_{s}(\theta, \omega)-q_{s}(\theta, \omega) \theta \geq 0 .
\end{aligned}
$$

With a standard analysis, an incentive compatible and individually rational mechanism yields the principal a payoff:

$$
V\left(\Gamma^{s}\right)=\int_{\Omega} \int_{\Theta}\left[v\left(\omega, q_{s}(\theta, \omega)\right)-q_{s}(\theta, \omega) \phi(\theta, \omega)\right] f(\theta \mid \omega) d \theta d G(\omega),
$$

where $\phi(\theta, \omega)$ is the virtual cost defined as $\phi(\theta, \omega) \equiv \theta+\frac{F(\theta \mid \omega)}{f(\theta \mid \omega)}$, and note that, from the conditions in the model, $\phi$ is a strictly increasing function of marginal cost $\theta$ but a strictly decreasing function of quality $\omega$. Under the pointwise maximization, the principal chooses $q_{s}$ to maximize the term with the virtual cost inside the integral in (7) in order to satisfy the incentive compatibility (5), not the total surplus $v(\omega, q)-\theta q$, the sum of (1) and (2), and its maximum value is denoted by $V_{s}$.

Then, the principal's second-best payoff from the benchmark is $V_{s}-\bar{U}-c$, and it can be readily shown that $V_{s}$ is higher than the partial contracting's optimal payoff $V_{p}$.

Proposition 2 Suppose that the principal can observe the quality. Then, the second-best optimal allocation is given as

$$
\begin{aligned}
& \text { (i) if } v_{q}(\omega, 0)-\phi(\theta, \omega) \leq 0, q_{s}(\theta, \omega)=0 \\
& \text { (ii) otherwise, } v_{q}\left(\omega, q_{s}(\theta, \omega)\right)-\phi(\theta, \omega)=0
\end{aligned}
$$

which yields $V_{s}$ higher than $V_{p}$. Hence, if $V_{s}-\bar{U}-c>V_{p}$, the principal acquires information on worker quality.

For interior solutions in (8), the conditions on $v$ and the distribution in the previous section, naturally, imply that the agent's production function $q_{s}(\theta, \omega)$ is a strictly decreasing function of marginal cost $\theta$ but a strictly increasing function of quality $\omega .^{25}$ The maximized term inside the integral in (7), by substituting the optimal $q_{s}(\theta, \omega)$ from Proposition 2, is denoted by $V(\theta, \omega)$ such that

$$
V(\theta, \omega) \equiv v\left(\omega, q_{s}(\theta, \omega)\right)-q_{s}(\theta, \omega) \phi(\theta, \omega) .
$$

With $V(\theta, \omega), V_{s}$ can be neatly written as $V_{s}=\int_{\Omega} \int_{\Theta} V(\theta, \omega) f(\theta \mid \omega) d \theta d G(\omega)$. The proof for $V_{s}>V_{p}$ simply confirms our intuition based on the difference in the principal's observability of the agent's quality $\omega$ : The maximization of the benchmark case with observable $\omega$ requires that for a higher value of $\omega$, the agent must produce more, whereas it is constant for the partial contracting. Then for $\bar{U}$ satisfying $\bar{U}<V_{s}-V_{p}$, if $c>0$ is sufficiently small, we have $V_{s}-\bar{U}-c>V_{p}$; the second best yields a higher payoff to the principal than the partial contracting. Hence, this result characterizes the condition under which the principal hires the manager in an attempt to achieve the second best if he cannot observe the quality; he would not have an incentive to do so at any cost otherwise. Thus, $V_{s}-\bar{U}-V_{p}>0$ is necessary for information acquisition, which is maintained in what follows.

\footnotetext{
${ }^{25}$ For each $\omega$, one obtains analogous results with the nonlinear pricing, including the "no distortion at the top and the downward distortion below the top" such that the agent with the lowest marginal cost $\underline{\theta}$ chooses the first-best quantity, whereas the agent with a marginal cost greater than $\underline{\theta}$ chooses a quantity lower than the first best.
} 
To illustrate the second-best solution, consider the example from the introduction with particular functional forms and one worker.

Example 1 A firm's owner assigns different working hours to a worker for a job among the total working hours $T>0$. The job's outcome depends on the worker's quality, and his cost function is $\theta q$ for $q \geq 0$ hours. The conditional distribution of cost $\theta$ given quality $\omega$ is $F(\theta \mid \omega)=\theta^{\omega}$ for $\theta \in[0,1]$, which yields the virtual cost $\phi(\theta, \omega)=\theta+\frac{\theta}{\omega}$. The principal's payoff from the job is $\omega \ln (q+1)$ if the worker with quality $\omega$ invests $q$ hours. The worker can be assigned to the other standard job for the remaining hours $T-q$, which gives the principal payoff $(T-q)$. Together, the principal obtains a total value $v(\omega, q)=\omega \ln (q+1)+(T-q)$. The distribution $F$ and $v$ satisfy the assumptions in Section 2, including the reverse hazard rate dominance. Additionally, the conditions for an interior output require $1<\underline{\omega}<\bar{\omega} \leq 2$. Then, the second-best solution from (8) is given as $q_{s}(\theta, \omega)=\frac{\omega^{2}}{(\theta \omega+\theta+\omega)}-1$.

\section{Optimal contracts}

In this section, we aim to find two different optimal mechanisms with the manager. A collection of mechanisms with the manager can be classified into two categories: selling the project to the manager, called ex-ante contracts, and those with the principal incentivizing the manager to acquire information on worker quality, simultaneous contracts. The sale of the project is not a subclass of the latter because, by selling it, the principal does not have to incentivize the manager to do so, unlike simultaneous contracts. We suppose, for this section, that the information cost $c>0$ is sufficiently small so that the manager always has such an incentive, as will be shown below, and defer further analysis on the role of the cost in shaping the optimal organizational structure to the next section.

One can argue that selling in fact provides the incentive for information acquisition as well; thus, it is a particular class of simultaneous contracting. That is, after buying the project, the manager himself finds it optimal to acquire the information if the condition $V_{s}-\bar{U}-c>V_{p}$ is satisfied, as discussed in the previous section. The ex-ante contracting, however, is constrained by limited liability in a direct manner, as shown below. On the other hand, the virtue of the simultaneous contracting's first-order alignment is to remove such direct effect of limited liability on the principal's payoff. Yet, the incentive alignment is also associated with the manager's payoff if he deviates. In essence, the interplay between the extent to which limited liability constrains the principal and the size of the information cost determines whether the simultaneous contracting or the ex-ante contracting is optimal. In that sense, more specifically, the simultaneous contracting mechanism refers to the one in which the principal's contract embodies the incentive for information acquisition.

\subsection{Ex-ante contracts: selling the project}

We find the optimal ex-ante contract first. After buying the project from the principal, the manager, as an informed mechanism designer, obtains $V_{s}$ like the benchmark, but he is constrained by limited liability unlike the principal. An ex ante contracting mechanism is said to be individually rational if $V_{s}-c-\alpha \geq \bar{U}$, where $\alpha$ denotes a sale price. It satisfies limited liability given $\alpha$ if $V(\theta, \omega)-c-\alpha \geq B$ 


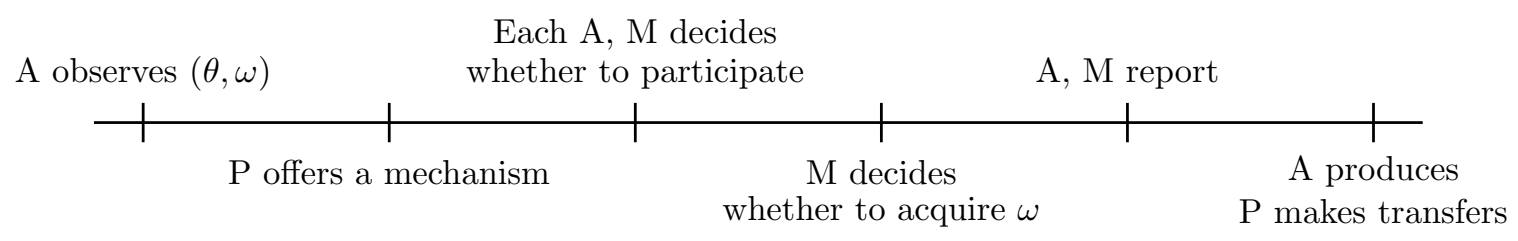

Figure 1: Timeline

for all $(\theta, \omega) \in \Theta \times \Omega$; or $\min _{(\theta, \omega)} V(\theta, \omega)-c-\alpha \geq B$, where $V(\theta, \omega)$ is the manager's (ex-post) optimal payoff, previously derived in (9). If the limited liability is "effective" in the sense that $V_{s}-\bar{U}>\min _{(\theta, \omega)} V(\theta, \omega)-B$, the ex ante contracting cannot achieve the second best payoff. ${ }^{26}$ Then, the optimal sale price from the ex ante contracting is

$$
\alpha=\min \left\{V_{s}-\bar{U}, \min _{(\theta, \omega)} V(\theta, \omega)-B\right\}-c .
$$

Depending on a condition identified in the result below, the optimal ex ante contracting payoff is strictly lower than the second-best for all $\bar{U}<V_{s}-V_{p}$, or for some $\bar{U}<V_{s}-V_{p}$, where recall the necessary condition for information acquisition $V_{s}-\bar{U}-V_{p}>0$ from Proposition 2 .

Proposition 3 The optimal ex ante contracting payoff is strictly dominated by the second-best payoff $V_{s}-\bar{U}-c$ if $V_{p} \geq \min _{(\theta, \omega)} V(\theta, \omega)-B$, and it is weakly dominated by the second-best payoff otherwise.

\subsection{Simultaneous contracting}

Now, with no selling, to extract worker quality, the principal designs a mechanism that simultaneously contracts with both the manager and the agent. The game's timeline after Nature chooses a type $(\theta, \omega)$ is shown in Figure 1.

The essential feature of the manager's contract is to provide incentive compatibility while satisfying both an incentive for information acquisition and limited liability. There are simple contracts that satisfy incentive compatibility together with either condition, partially. On one hand, a constant payment and punishment for two different reports satisfy incentive compatibility and limited liability. Unlike the standard case in which the principal uses either form of contracts only for truthful incentive compatibility, the principal of this model must also incentivize information acquisition. That is, he must handle combinatorial deviations - the manager's misreport of quality following no information acquisition. However, for those simple contracts, the information cost makes an optimal or equilibrium payoff without acquiring the information greater than that with acquiring it. On the other hand, the ex-ante contracts can provide the incentive for information acquisition, but its direct negative effects are discussed previously.

\footnotetext{
${ }^{26} \mathrm{It}$ is not determinant whether the limited liability is effective or not, since, in spite of $\min (\theta, \omega) q(\theta, \omega)=0$, we may have $\min _{(\theta, \omega)} V(\theta, \omega)>0$; for instance, in Example 1, it is $\min _{(\theta, \omega)} V(\theta, \omega)=T>0$, and $B$ can take a negative value. The principal cannot make the manager's contract contingent on the true quality $\omega$ or $V(\theta, \omega)$, but, somewhat beyond the scope of this model, he might sell a "seat" to invite the manager as a co-principal; still, the limited liability can be effective, that is, $\frac{1}{2} V_{s}-\bar{U}>\frac{1}{2} V_{\min }-B$ and continue to be so as long as the number of seats is bounded.
} 
We connect the manager's contract with the principal's optimality through the first-order condition to tackle limited liability, and this "task" has to be done while providing the incentive for information acquisition. To incentivize information acquisition via the correlation between the two dimensions of a type, the principal elicit worker quality $\omega$ only from the manager. ${ }^{27}$. Any contract for the manager that utilizes such correlation must make it contingent on the agent's quantity $q_{s}(\theta, \omega)$, so it is related to Crémer and McLean (1988), but this incentive alignment differs from them in that the alignment applies correlation to the first-order condition in order to decouple any base compensation for limited liability from it providing incentive compatibility. ${ }^{28}$

Then, a direct mechanism $\Gamma$ consists of two-dimensional functions $q$, $t$, and $S$, where $q: \Theta \times \Omega \rightarrow$ $\mathbb{R}_{+}, t: \Theta \times \Omega \rightarrow \mathbb{R}$ and $S: \Omega \times \Theta \rightarrow \mathbb{R}$. If the agent reports $\theta$ and the manager reports $\omega$, then the principal assigns the agent the production of $q(\theta, \omega)$ and commits to paying $t(\theta, \omega)$ to the agent and $S(\omega, \theta)$ to the manager. A contract for the manager $S$ is said to be incentive compatible (IC) if for each $\omega, \omega^{\prime} \in \Omega$,

$$
\mathbb{E}_{\theta}[S(\omega, \theta) \mid \omega] \geq \mathbb{E}_{\theta}\left[S\left(\omega^{\prime}, \theta\right) \mid \omega\right]
$$

Like the agent's payoff in (1), the (true) quality $\omega$ does not affect the manager's payoff $S\left(\omega^{\prime}, \theta\right)$; that is, it affects neither the manager's "direct" payoff nor the agent's. However, the quality $\omega$ does affect the manager's expected payoff through the conditional distribution of $\theta$ given $\omega$. A contract for the manager is individually rational (IR) if

$$
\int_{\Omega} \mathbb{E}_{\theta}[S(\omega, \theta) \mid \omega] d G(\omega)-c \geq \bar{U}
$$

and it satisfies limited liability (LL) if for each $\omega \in \Omega$ and every $\theta \in \Theta$,

$$
S(\omega, \theta)-c \geq B
$$

If the manager does not acquire the information, then he chooses a report $\widehat{\omega}$ maximizing his ex-ante payoff without incurring the cost $c$. A contract for the manager incentivizes information acquisition (IA) if for each $\widehat{\omega} \in \Omega$,

$$
\int_{\Omega} \mathbb{E}_{\theta}[S(\omega, \theta) \mid \omega] d G(\omega)-c \geq \int_{\Omega} \mathbb{E}_{\theta}[S(\widehat{\omega}, \theta) \mid \omega] d G(\omega) .
$$

IA is not satisfied unless IC is satisfied with strict inequality over a measurable set of $\omega$, as, for example, a constant payment.

The principal obtains his expected payoff from a mechanism $\Gamma$ such that

$$
V(\Gamma)=\int_{\Omega} \int_{\Theta}[v(\omega, q(\theta, \omega))-t(\theta, \omega)-S(\omega, \theta)] f(\theta \mid \omega) d \theta d G(\omega) .
$$

Unlike the payoff from the second best in (7), now, the principal pays the manager $S(\omega, \theta)$ to make him acquire information on the quality. Once the conditions for the manager's contract $S$, (11)

\footnotetext{
${ }^{27}$ Otherwise, as separate issues, we can have multiplicity problem and collusion. We relegate related discussions to Appendix B.1.

${ }^{28}$ The literature that extended Crémer and McLean (1988) based on their Theorem 1 adopts "lotteries" only for the rent extraction, while incentive compatibility is provided by a VCG mechanism. However, the VCG for the manager of this model (any third party as in Tirole (1986)) is a constant payment that fails to provide IA.
} 
- (14), are satisfied, any direct mechanism $(q, t, S)$ implementing the second-best payoff must be $\left(q_{s}, t_{s}, S\right)$, where $\left(q_{s}, t_{s}\right)$ is the optimal choice from the second-best solution in Proposition 2, which makes the truthful report of $\theta$ the agent's weakly dominant strategy (i.e., the agent's IC \& IR hold). Then, a simultaneous contracting mechanism $\Gamma$ is optimal if it maximizes the above payoff $V(\Gamma)$ subject to IC, IR, LL and IA, with the optimal contract for the agent $\left(q_{s}, t_{s}\right)$.

The manager's payoff is only based on the monetary compensation from the principal, as in Tirole (1986) as well as other papers following it; i.e., $S\left(\omega^{\prime}, \theta\right)$ is both the manager's contract and his payoff. Hence, we use instead the principal's payoff function and his first-order condition to solve the delicate problem - providing IC together with IA and LL. In fact, the principal's first-order condition is the only such optimality condition available.

The first main result establishes that under IC, a contract for the manager satisfies the principal's first-order optimality if and only if it has an interval structure. The principal's first-order condition under pointwise maximization can be found from Proposition 2, and thereby consider the following: if $\omega^{\prime}=\omega \in \Omega$,

$$
v_{q}\left(\omega^{\prime}, q_{s}\left(\theta, \omega^{\prime}\right)\right)-\phi(\theta, \omega)=0 \text { satisfying IC. }
$$

If the principal can utilize the condition above for the manager's contract to satisfy IC, the equality means that if the manager reports the quality truthfully, i.e., $\omega^{\prime}=\omega$, then the manager's firstorder optimality is satisfied. Obviously, not all such contracts from $v_{q}\left(\omega^{\prime}, q_{s}\left(\theta, \omega^{\prime}\right)\right)-\phi(\theta, \omega)=0$ satisfy IC; that is, a collection of incentive compatible (IC) contracts among contracts satisfying the above first-order condition is a subset of a set of contracts satisfying only the condition, the derivative being equal to zero. For example, the lowest cost type $\underline{\theta}$ is to be excluded to make IC hold since otherwise, the true quality $\omega$ in $\phi(\theta, \omega) \equiv \theta+\frac{F(\theta \mid \omega)}{f(\theta \mid \omega)}$ simply disappears, and even then, the condition is only necessary for truth-telling. We term the way of relating the two optimality conditions the first-order alignment because the manager's contract is aligned with the principal's optimality through the first-order condition.

On the other hand, a contract for the manager $S\left(\omega^{\prime}, \theta\right)$ is said to have an interval structure if it yields the expected payoff such as

$$
\underline{S}+s\left(\omega^{\prime}, q\right) \operatorname{Pr}\left(q_{s}\left(\theta, \omega^{\prime}\right)>q \mid \omega\right) \text { satisfying IC, }
$$

where $\underline{S}$ is a constant for the base compensation handling LL, which disappears via the decoupling. The interpretation is justified since the most simple and intuitive contract to generate such a payoff in $(17)$ is

$$
S\left(\omega^{\prime}, \theta\right)= \begin{cases}\underline{S}+s\left(\omega^{\prime}, q\right) & \text { if } q_{s}\left(\theta, \omega^{\prime}\right)>q, \\ \underline{S} & \text { otherwise. }\end{cases}
$$

That is, the principal pays the manager $s\left(\omega^{\prime}, q\right)$ for his report $\omega^{\prime}$ if the agent's quantity $q_{s}\left(\theta, \omega^{\prime}\right)$ turns out to be greater than a "target" quantity $q .{ }^{29}$ Moreover, to make the contracts generating $\underline{S}+s\left(\omega^{\prime}, q\right) \operatorname{Pr}\left(q_{s}\left(\theta, \omega^{\prime}\right)>q \mid \omega\right)$ satisfy IC, by the reverse hazard rate dominance, a condition like the

\footnotetext{
${ }^{29}$ There can be many other forms that can yield (21); for instance, the principal pays the manager $s\left(\omega^{\prime}, q\right)+a\left(\omega^{\prime}, q\right)$ if $q_{s}\left(\theta, \omega^{\prime}\right)>q$, and pays him $b\left(\omega^{\prime}, q\right)$ otherwise, which yields him the same expected payoff in (17) as long as $\operatorname{Pr}\left(q_{s}\left(\theta, \omega^{\prime}\right)>q \mid \omega\right)\left[a\left(\omega^{\prime}, q\right)-b\left(\omega^{\prime}, q\right)\right]+b\left(\omega^{\prime}, q\right)=0$. As in any mechanism design problem, we have (17) as well as (16), given the agent's truthful report by the contract for him $\left(q_{s}, t_{s}\right)$, the optimal choice from Proposition 2.
} 


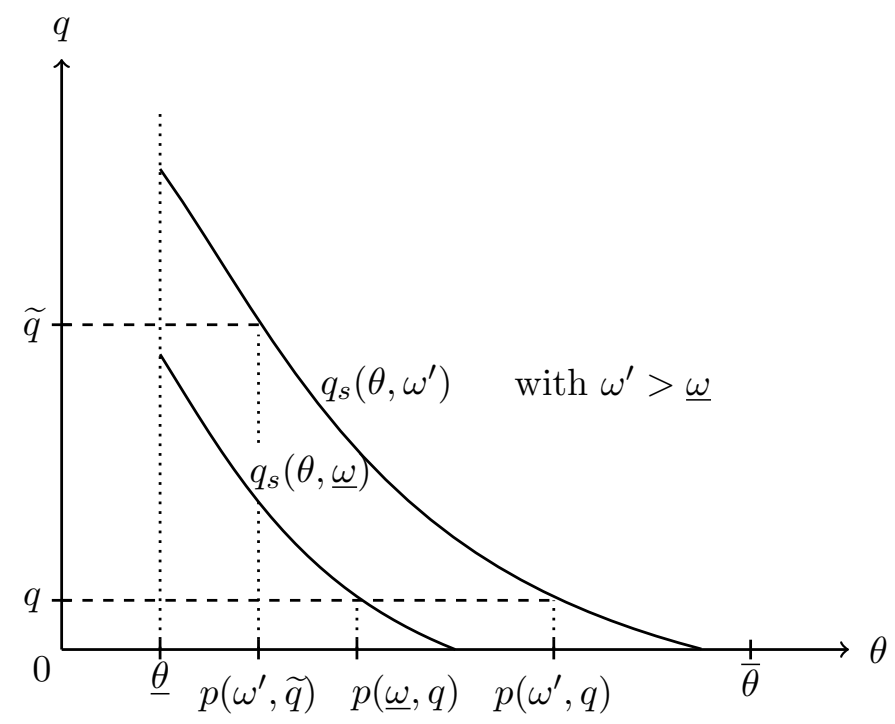

Figure 2: $q_{s}$ and $p$ mappings

single-crossing property between the manager's report $\omega^{\prime}$ and the true $\omega$ is endogenously created in his conditional expected payoff in (17) (see the full-fledged proof of Theorem 1 in Appendix).

We first show that any contract for the manager satisfying the principal's first-order condition comes with such a structure - that is, $(16) \Rightarrow(17)$ - as it is simple and illuminating. The other implication, $(16) \Leftarrow(17)$, can be shown after a Lemma for differentiability is established. We reformulate the optimality in (16) with a cost type $\theta=p\left(\omega^{\prime}, q\right)$ (its formal definition is given in Lemma 1 taking care of $q=0$ ) satisfying

$$
q_{s}\left(p\left(\omega^{\prime}, q\right), \omega^{\prime}\right)=q
$$

For $q>0, p\left(\omega^{\prime}, q\right)$ is the cost that produces $q$ given quality $\omega^{\prime}$, and it is simply an inverse function of $q_{s}\left(\theta, \omega^{\prime}\right)$ with respect to $q$ and $\theta$, holding $\omega^{\prime}$ fixed. Note that $q_{s}\left(\theta, \omega^{\prime}\right)>q \Leftrightarrow \theta<p\left(\omega^{\prime}, q\right)$, where their inverse relationship is depicted in Figure 2. With (19), the first-order condition in (16) can be rewritten such that if $\omega^{\prime}=\omega \in \Omega$,

$$
v_{q}\left(\omega^{\prime}, q\right)-\phi\left(p\left(\omega^{\prime}, q\right), \omega\right)=0 \text { satisfying IC. }
$$

The qualification $\theta>\underline{\theta}$ from (16) for IC is translated to $q=q_{s}(\theta, \omega)$ for $\theta>\underline{\theta}$.

After incorporating $\phi(\theta, \omega)$, we factor out $p_{\omega}\left(\omega^{\prime}, q\right)$, the partial derivative of $p\left(\omega^{\prime}, q\right)$ with respect to $\omega^{\prime}$. Then, by a simple algebra, as shown in the proof of Theorem 1 and Appendix B.2, the above (20) becomes: under IC,

$$
\frac{\partial\left[\log \left(e\left(\omega^{\prime}, q\right) F\left(p\left(\omega^{\prime}, q\right) \mid \omega\right)\right)\right]}{\partial \omega^{\prime}}=0 \text { if } \omega^{\prime}=\omega \in \Omega,
$$

where $e(\omega, q)$ function is uniquely determined as

$$
e(\omega, q) \equiv \exp \left\{\int_{\omega}^{\bar{\omega}} \frac{p_{\omega}(x, q)}{v_{q}(x, q)-p(x, q)} d x\right\} .
$$


Since the first-order condition (21) is necessary, the way of integrating (22) provides the sufficiency for IC. The solution $e\left(\omega^{\prime}, q\right)$ is scale free, so we can always put a constant $\bar{s}>0$ on $e(\omega, q)$ to have $s\left(\omega^{\prime}, q\right)=\bar{s} e\left(\omega^{\prime}, q\right) .{ }^{30}$ Then, given that log is increasing, $\omega^{\prime}$ solves max $s\left(\omega^{\prime}, q\right) F\left(p\left(\omega^{\prime}, q\right) \mid \omega\right)$ in $(17)$ if and only if it solves the max of logarithm of it. Hence, if $(20)$ holds, $\partial u\left(\omega^{\prime}, \omega, q\right) / \partial \omega^{\prime}=0$ for all $\omega^{\prime}=\omega \in \Omega$ satisfying IC, so $(16) \Rightarrow(17)$.

Before embarking on the proof for $(16) \Leftarrow(17)$, we consider one natural class of interval structures extending (17): multiple target levels, not just one. Further, this enables us to pursue the question of choosing target quantity levels endogenously in the next section. Yet, we restrict a number of target quantity levels to be finite based on the following two reasonings: First, one ought to consider how plausible to have an infinite (or continuum) number of target levels in the real world sense, and second, solving the endogenous target quantity levels with an infinite set becomes an infinite dimensional problem. For the generalization, it is convenient to use notation $q_{i}$ for target $i$ and also denote $s_{i}\left(\omega^{\prime}\right) \equiv s\left(\omega^{\prime}, q_{i}\right)=\bar{s}_{i} e\left(\omega^{\prime}, q_{i}\right)$. Then, for any finite number of target quantity levels with $q_{0}<\cdots<q_{N-1}<q_{s}(\underline{\theta}, \underline{\omega})$, where $q_{i}<q_{s}(\underline{\theta}, \underline{\omega})$ is from $q=q_{s}(\theta, \omega)$ for $\theta>\underline{\theta}$ in (16), ${ }^{31}$ the payoff in (17) is expanded such as

$$
\mathbb{E}_{\theta}\left[S\left(\omega^{\prime}, \theta\right) \mid \omega\right]=\underline{S}+\sum_{i=0}^{N-1} u\left(\omega^{\prime}, \omega, q_{i}\right),
$$

where denote $u\left(\omega^{\prime}, \omega, q_{i}\right) \equiv s_{i}\left(\omega^{\prime}\right) \operatorname{Pr}\left(q_{s}\left(\theta, \omega^{\prime}\right)>q_{i} \mid \omega\right)$.

In deriving the contract in (17) from (16), we can find a couple of nice properties for its solution $s_{i}\left(\omega^{\prime}\right)=\bar{s}_{i} e\left(\omega^{\prime}, q_{i}\right)$ where $e\left(\omega^{\prime}, q_{i}\right)$ is from (22). First, $s_{i}\left(\omega^{\prime}\right)>0$ and $F\left(p\left(\omega^{\prime}, q_{i}\right) \mid \omega\right)>0$ for all $\omega, \omega^{\prime} \in \Omega$ from $q_{i}<q_{s}(\underline{\theta}, \underline{\omega})$, and $s_{i}\left(\omega^{\prime}\right)$ is positive, differentiable and strictly decreasing on $\Omega$. Now, we start with an interval structure (23), extending (17), to arrive at (16). This means that we need to prove those properties from (23).

Lemma 1 For any finite number of target quantity levels in (23), if $S(\theta, \omega)$ satisfies $I C$, each $q_{i}$ satisfies the following properties.

(i) $s_{i}\left(\omega^{\prime}\right) F\left(p\left(\omega^{\prime}, q_{i}\right) \mid \omega\right)>0$ for all $\omega, \omega^{\prime} \in \Omega$, where $p\left(\omega^{\prime}, q_{i}\right) \equiv \min \left\{\theta \in[\underline{\theta}, \bar{\theta}]: q_{s}\left(\theta, \omega^{\prime}\right)=q_{i}\right\}$.

(ii) $s_{i}\left(\omega^{\prime}\right)$ is differentiable and strictly decreasing on $\Omega$.

The first result of the Lemma implies that $s_{i}\left(\omega^{\prime}\right)>0$ and $F\left(p\left(\omega^{\prime}, q_{i}\right) \mid \omega\right)>0$ for all $\omega, \omega^{\prime} \in \Omega$. If the former is violated, IC has a conflict with the reverse hazard rate dominance. The latter requires a target quantity to yield a positive probability for any report. If it is violated, the manager can misreport a quality level that can give him a positive expected payoff. Additionally, $s_{i}\left(\omega^{\prime}\right)$ has a negative relationship with the manager's report on worker quality: a "penalty" on the manager's over-reporting incentive of quality for higher output. Together, the intuition behind the result is

\footnotetext{
${ }^{30}$ That is, the fraction of the first order derivative of $s\left(\omega^{\prime}, q\right)$ with respect to $\omega^{\prime}$ and $s\left(\omega^{\prime}, q\right)$ can be expressed as one functional form, i.e., $\frac{\partial s\left(\omega^{\prime}, q\right) / \partial \omega^{\prime}}{s\left(\omega^{\prime}, q\right)}=y\left(\omega^{\prime}, q\right)$. This is known as the first-order homogeneous differential equation, which is invariant under changes of scale.

${ }^{31}$ Note " $q=q_{s}\left(\theta, \omega^{\prime}\right)$ given all $\theta>\underline{\theta}$ for all $\omega^{\prime \prime}$ " is equivalent to $q<q_{s}(\underline{\theta}, \underline{\omega})$. To understand it, if the target quantity is chosen high, for instance, $\widetilde{q}$ in Figure 2, for $\underline{\omega}$, there is simply no cost type that can produce it, which violates a necessary condition for IC.
} 

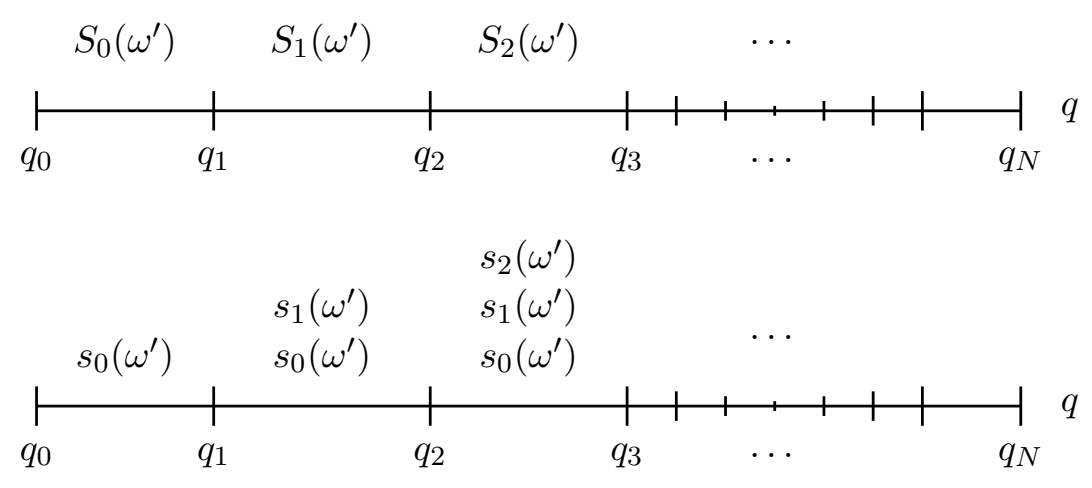

Figure 3: A compensation scheme

that if the agent's output meets a target, the manager is rewarded with a positive payoff but the magnitude itself is strictly decreasing in his report on the quality.

Theorem 1 establishes the equivalence between the first-order alignment and an interval structure, and the contract is unique up to a positive linear affine transformation. Furthermore, the principal always attains the second-best payoff, $V_{s}-\bar{U}-c$ for a low information cost $c>0$; the mechanism dominates the optimal ex ante contracting. ${ }^{32}$

Theorem 1 Under the manager's incentive compatibility (11), a contract for the manager satisfies the principal's first-order optimality if and only if it has an interval structure for any finite number of target quantity levels with $q_{0}<\cdots<q_{N-1}<q_{s}(\underline{\theta}, \underline{\omega})$. The manager's contract is unique up to a positive affine transformation such that for each $i$, its compensation $s_{i}(\omega)$ is given as

$$
s_{i}(\omega)=\bar{s}_{i} e\left(\omega, q_{i}\right),
$$

where $\bar{s}_{i}>0$ and $e$ is defined by (22). By choosing the base compensation $\underline{S}=B+c$, the optimal simultaneous contracting mechanism makes the manager's individual rationality (12) binding, while satisfying the limited liability (13), which dominates the optimal ex ante contracting mechanism. If the information cost is sufficiently small, this contract incentivizes information acquisition (14).

The contract yields the strict IC. ${ }^{33}$ In addition, a collection of $\left(s_{i}\left(\omega^{\prime}\right)\right)_{i=0, \ldots, N-1} \in \mathbb{R}^{N}$ can be reinterpreted as a collection of the manager's payoffs $S_{i}\left(\omega^{\prime}\right) \geq 0$ for each $i=0,1, \ldots, N-1$ such that

$$
S_{i}\left(\omega^{\prime}\right) \equiv \begin{cases}s_{i}\left(\omega^{\prime}\right) & \text { if } i=0 \\ S_{i-1}\left(\omega^{\prime}\right)+s_{i}\left(\omega^{\prime}\right) & \text { if } i \geq 1\end{cases}
$$

In that regard, a collection of $\left(s_{i}\left(\omega^{\prime}\right)\right)_{i=0, \ldots, N-1} \in \mathbb{R}^{N}$ can be interpreted as a collection of marginal compensation payments. In other words, the contract with $\left(s_{i}\left(\omega^{\prime}\right)\right)_{i=0, \ldots, N-1} \in \mathbb{R}^{N}$ for target quantity levels can be equivalently expressed as a finite number of intervals such that

$$
S\left(\omega^{\prime}, \theta\right)= \begin{cases}\underline{S} & \text { if } q_{s}\left(\theta, \omega^{\prime}\right)=0 \\ S_{i}\left(\omega^{\prime}\right) & \text { if } q_{s}\left(\theta, \omega^{\prime}\right) \in\left[q_{i}, q_{i+1}\right] \text { and } q_{s}\left(\theta, \omega^{\prime}\right) \neq 0\end{cases}
$$

\footnotetext{
${ }^{32}$ It dominates the optimal ex ante mechanism weakly or strongly depending on the condition identified in Proposition 3 .

${ }^{33}$ That is, $u_{\omega^{\prime}}\left(\omega^{\prime}, \omega, q_{i}\right)>0$ for all $\omega^{\prime}<\omega$, and $u_{\omega^{\prime}}\left(\omega^{\prime}, \omega, q_{i}\right)<0$ for all $\omega^{\prime}>\omega$.
} 
where the equivalence between them is illustrated in Figure 3. In particular, Lemma 1 shows that the incentive compatible contract with intervals is monotone, $S_{0}\left(\omega^{\prime}\right)<S_{1}\left(\omega^{\prime}\right)<\cdots<S_{N-1}\left(\omega^{\prime}\right)$. Thus, the intuition of the Lemma expands: The principal rewards the manager more if the agent's quantity passes an additional target level, but each positive magnitude is strictly decreasing in the manager's report on the quality.

\subsection{Payoff structure}

The uniqueness characterization yields a linear structure for $\int_{\Omega} \mathbb{E}_{\theta}[S(\omega, \theta) \mid \omega] d G(\omega)$, the expectation of $\mathbb{E}_{\theta}[S(\omega, \theta) \mid \omega]$ in $(23)$, with respect to $\bar{s} \equiv\left(\bar{s}_{0}, \ldots, \bar{s}_{N-1}\right)$ from Theorem 1 such that

$$
\int_{\Omega} \mathbb{E}_{\theta}[S(\omega, \theta) \mid \omega] d G(\omega)=\underline{S}+\sum_{i=0}^{N-1} \bar{s}_{i} U\left(q_{i}\right),
$$

where we denote $U\left(q_{i}\right) \equiv \int_{\Omega} e\left(\omega, q_{i}\right) F\left(p\left(\omega, q_{i}\right) \mid \omega\right) d G(\omega)$. On the other hand, if the manager maximizes his ex-ante payoff without acquiring the information, his payoff from reporting $\widehat{\omega}$ is

$$
\int_{\Omega} \mathbb{E}_{\theta}[S(\widehat{\omega}, \theta) \mid \omega] d G(\omega)=\underline{S}+\sum_{i=0}^{N-1} \bar{s}_{i} \widehat{U}\left(\widehat{\omega}, q_{i}\right),
$$

where denote $\widehat{U}\left(\widehat{\omega}, q_{i}\right) \equiv e\left(\widehat{\omega}, q_{i}\right) \int_{\Omega} F\left(p\left(\widehat{\omega}, q_{i}\right) \mid \omega\right) d G(\omega)$. Hence, $\widehat{U}\left(\widehat{\omega}, q_{i}\right)$ is a unit-weight $\left(\bar{s}_{i}=1\right)$ ex-ante payoff from a misreport $\widehat{\omega}$ without acquiring $\omega \in \Omega$, whereas $U\left(q_{i}\right)$ is a unit-weight ex-ante payoff from truthful reports after acquiring it for all $\omega \in \Omega$. With them, the binding IR yields $\left[\underline{S}+\sum_{i=0}^{N-1} \bar{s}_{i} U\left(q_{i}\right)\right]-c=\bar{U}$, while IA requires $\sum_{i=0}^{N-1} \bar{s}_{i} U\left(q_{i}\right)-c \geq \sum_{i=0}^{N-1} \bar{s}_{i} \widehat{U}\left(\widehat{\omega}, q_{i}\right)$ for all $\widehat{\omega} \in \Omega$.

Note that IA is satisfied for different target quantity levels or partitions if the information cost $c$ is small because for each $i$, by the strict IC from the incentive alignment, we have $U\left(q_{i}\right)>$ $\widehat{U}\left(\widehat{\omega}, q_{i}\right)$ for all $\widehat{\omega} \cdot{ }^{34}$ However, if the information acquisition is costly, choosing an interval structure or a partition to satisfy IA is a delicate problem; eventually, it requires the principal to choose $\left(\bar{s}_{0}, \ldots, \bar{s}_{N-1}\right)$ to minimize $\max _{\widehat{\omega}} \sum_{i=0}^{N-1} \bar{s}_{i} \widehat{U}\left(\widehat{\omega}, q_{i}\right)$ to find out the maximum cost to satisfy IA, as we will study in the next section.

\section{Information Acquisition}

In the previous section, if the information acquisition is not costly, the compensation $S(\theta, \omega)$ for the manager in the optimal simultaneous contract extracts all possible rent from him, neglecting IA. However, if the cost $c>0$ is not negligible, choosing an interval structure with target levels demands careful treatment. Relatedly, for a high information cost, instead of the full extraction, it may be optimal for the principal to leave some surplus to the manager and thereby incentivize him to still acquire the costly information. The trade-off leads to a firm's boundary next section.

\footnotetext{
${ }^{34}$ Since the information cost $c>0$ is sufficiently small, neglecting IA, we may choose a vector of normalized weights following $\lambda_{i} \equiv \frac{\bar{s}_{i}}{\sum_{i=0}^{N-1} \bar{s}_{i}}>0$ to illustrate how the linear transformation can bind IR. From the binding IR with the normalized weights $\left(\lambda_{0}, \ldots, \lambda_{N-1}\right),\left[B+c+\left(\sum_{i=0}^{N-1} \bar{s}_{i}\right) \sum_{i=0}^{N-1} \lambda_{i} U\left(q_{i}\right)\right]-c=\bar{U}$, we can show that different weights $\left(\bar{s}_{0}, \ldots, \bar{s}_{N-1}\right)$ satisfy the binding IR and IA as long as $\sum_{i=0}^{N-1} \bar{s}_{i}=\frac{\bar{U}-B}{\sum_{i=0}^{N-1} \lambda_{i} U\left(q_{i}\right)}$.
} 
Prior to that, we study viability of the simultaneous contracting, which boils down to identifying the maximum information cost that permits it given a rent.

The trade-off between information acquisition and the manager's extra rent can be formalized by denote the rend by $R \geq 0$ with binding his IR; that is, $\int_{\Omega} \mathbb{E}_{\theta}[S(\omega, \theta) \mid \omega] d G(\omega)-c=\bar{U}+R$, where recall $\int_{\Omega} \mathbb{E}_{\theta}[S(\omega, \theta) \mid \omega] d G(\omega)=\underline{S}+\sum_{i=0}^{N-1} \bar{s}_{i} U\left(q_{i}\right)$ in (25). The extra surplus $R$ is introduced since $R$ is a transfer, unlike $\bar{U}$, between the principal and the manager, with the connection to the next section - the boundary of the firm. Now, the binding IA in (14) yields the maximum information cost for a simultaneous contracting mechanism such that

$$
c=\int_{\Omega} \mathbb{E}_{\theta}[S(\omega, \theta) \mid \omega] d G(\omega)-\int_{\Omega} \mathbb{E}_{\theta}[S(\widehat{\omega}, \theta) \mid \omega] d G(\omega),
$$

where $\int_{\Omega} \mathbb{E}_{\theta}[S(\widehat{\omega}, \theta) \mid \omega] d G(\omega)=\underline{S}+\sum_{i=0}^{N-1} \bar{s}_{i} \widehat{U}\left(\widehat{\omega}, q_{i}\right)$ in $(26)$. Since any $\bar{s}_{i}$ can be chosen to be zero to make it ineffective, the problem of choosing both weights $\bar{s}=\left(\bar{s}_{0}, \ldots, \bar{s}_{N-1}\right)$ and intervals $q=\left(q_{0}, \ldots, q_{N-1}\right)$ can be reduced to the problem of choosing only weights $\bar{s}^{\prime}=\left(\bar{s}_{0}^{\prime}, \ldots, \bar{s}_{\bar{N}-1}^{\prime}\right)$ given the finest possible intervals $q^{\prime}=\left(q_{0}^{\prime}, \ldots, q_{\bar{N}-1}^{\prime}\right)$ with $\bar{N}$ intervals. In other words, finding optimal intervals as well as corresponding weights on them can be simplified as only determining optimal weights on all feasible intervals. We restrict a feasible scale to be finite to not have the problem with existence of a solution in infinite or continuum dimensions, as discussed, apart from reflecting the reality.

To find the maximum feasible information cost, with (25) and (26), we solve the following continuous minimax problem in $\bar{N}$ dimensions.

$$
\begin{aligned}
\underset{\bar{s} \in \mathbb{R}_{+}^{\bar{N}}}{\operatorname{minimize}} \max _{\widehat{\omega} \in \Omega} \underline{S}+\sum_{i=0}^{\bar{N}-1} \bar{s}_{i} \widehat{U}\left(\widehat{\omega}, q_{i}\right), \\
\text { subject to } \underline{S} \geq B+c, \\
{\left[\underline{S}+\sum_{i=0}^{\bar{N}-1} \bar{s}_{i} U\left(q_{i}\right)\right]-c=\bar{U}+R . }
\end{aligned}
$$

The principal finds an optimal interval structure to minimize the manager's deviation payoff, given the extra rent $R$, to identify the maximum admissible information cost. We show the existence of a minimax solution and further linearity of the minmaximized function in $R$.

Theorem 2 There exists a solution to the optimal interval structure problem (28), and the minmaximized function is linear in $R$.

With single-peak property of two intervals, we can further characterize that the optimal number of intervals is simply two and see Appendix B.3 for it. The solution to the minimax problem is critical to determining whether a simultaneous contracting is feasible or not for a non-negligible information cost $c$, and ultimately, a firm's limitation.

\section{Boundary of the Firm}

Even solving the minimax in the previous section - to minimize the deviation payoff and thereby maximize a feasible information cost - may fail to make the simultaneous contracting feasible. That 
is, the information cost can still be greater than the contracting's maximum possible information cost; minimizing the manager's deviation payoff may fail to overcome the cost of acquiring the information. Yet, this failure opens up an interesting question as to where one can draw a firm's boundary. Specifically, by incorporating the minimax problem's solution $S^{*}$ in (28) into the binding IA in (27), we find the threshold cost given an extra rent $R$ that admits a simultaneous contracting, which is denoted by $k(R)$ such that

$$
k(R) \equiv \int_{\Omega} \mathbb{E}_{\theta}\left[S^{*}(\omega, \theta) \mid \omega\right] d G(\omega)-\int_{\Omega} \mathbb{E}_{\theta}\left[S^{*}(\widehat{\omega}, \theta) \mid \omega\right] d G(\omega) .
$$

If the information cost $c$ is lower than $k(R)$ for an extra rent - the equality also satisfying IA - the principal can implement a simultaneous contracting as in Theorem 1 but with the rent $R$. If, on the other hand, it is greater than the threshold, the manager will not incur the cost to acquire the information, leaving the principal two options: ex-ante contracting or partial contracting (i.e., no contracting with the manager).

Table 1 encapsulates the three mechanisms studied so far with their corresponding optimal payoffs from Proposition 1 and Proposition 3 and Theorem 1 with the minimax in (28), where we denote $V_{\min } \equiv \min _{(\theta, \omega)} V(\theta, \omega)$. Given the trade-off between information acquisition and the

\begin{tabular}{l|l} 
Partial contracting & $V_{p}$ \\
\hline Ex ante contracting & $\min \left\{V_{s}-\bar{U}, V_{\min }-B\right\}-c$ \\
\hline Simultaneous contracting & $V_{s}-(\bar{U}+R)-c$
\end{tabular}

Table 1: Payoffs from three mechanisms

extra rent through the transfer $R$, the comparison between the simultaneous contracting and either mode of contracting with no information transmission first demands a simple characterization of the threshold admissible cost $k(R)$. Lemma 2 shows that $k(R)$ is in fact a linear and strictly increasing function of an extra rent $R$ such that

$$
k(R)=(\bar{U}-B+R)\left[1-\underset{x \in \Delta}{\operatorname{minimize}} \max _{\widehat{\omega} \in \Omega} \sum_{i=0}^{\bar{N}-1} x_{i} \frac{\widehat{U}\left(\widehat{\omega}, q_{i}\right)}{U\left(q_{i}\right)}\right],
$$

as the value inside bracket above is fixed once it is solved for $x \in \Delta$.

Then, with the simple linear function and Table 1, we compare the simultaneous contracting with the ex ante contracting. If limited liability is not "effective" in the sense that $V_{s}-\bar{U} \leq$ $V_{\text {min }}-B$, then for any positive rent $R>0$, the ex ante contracting, the selling, dominates the simultaneous contracting; there is no reason for the principal to incentivize the manager to acquire the information. ${ }^{35}$ If it is effective, $V_{s}-\bar{U}>V_{\min }-B$, on the other hand, the principal has an incentive to do so when $c \leq k(R)$ for $R<V_{s}-\bar{U}-\left(V_{\min }-B\right)$. Hence, if positive, we can define the difference between $\left(V_{s}-\bar{U}\right)$ and $\left(V_{\min }-B\right)$ as the maximum rent that the principal is willing to pay to the manager for information acquisition, compared with the ex ante contracting, and denote it by

$$
\Delta U \equiv \max \left\{V_{s}-\bar{U}-\left(V_{\min }-B\right), 0\right\}
$$

\footnotetext{
${ }^{35}$ Recall that $V_{\min }$ may be positive and $B$ may take a negative value.
} 
Since the main focus of this paper is to identify conditions under which the simultaneous contracting is the optimal organizational structure, we first consider the case that the necessary condition $\Delta U>0$ is satisfied; the other case $\Delta U=0$ will be treated later. The dominance of the simultaneous contracting over the partial contracting is less straightforward since the information cost is not cancelled out, unlike its dominance over the ex ante contracting.

Nevertheless, the simple functional form of $k(R)$ enables us to find $\widehat{R}$ such that the simultaneous contracting dominates either mode of contracting with no information transmission if $c<k(\widehat{R})$.

Lemma 2 Suppose the effective limited liability $\Delta U>0$. The threshold admissible cost $k(R)$ is derived as (30), so (i) for a contracting with no information transmission, there exists a threshold rent $\widehat{R}>0$ such that the simultaneous contracting dominates it if $c<k(\widehat{R})$ such that

$$
\widehat{R}= \begin{cases}\Delta U & \text { for the ex ante contracting } \\ V_{s}-\bar{U}-V_{p}-\widehat{c} & \text { for the partial contracting }\end{cases}
$$

where $\widehat{c}$ satisfies $\widehat{c}=k\left(V_{s}-\bar{U}-V_{p}-\widehat{c}\right)$; and (ii) for $c<k(\widehat{R})$, the optimal rent is $R=0$ if $c \leq k(0)$; $R=k^{-1}(c)$ if $k(0)<c<k(\widehat{R})$.

Lemma 2 also expands the concept of dominance of the simultaneous contracting in Theorem 1 such that even for a non-negligible information cost, there exists a corresponding unique $R$ to make the simultaneous contracting still dominant. That is, by the lemma, we can quantify how small is sufficient in Theorem 1's "sufficiently small" cost, which is $c \leq k(0)$, and further, the simultaneous contracting can dominate even for $c>k(0)$ by paying $R>0$ to minimally compensate the cost $c$, i.e., $R=k^{-1}(c)$, though it cannot achieve the second best.

Since the result above is written for a pairwise comparison with respect to the simultaneous contracting, determining the optimal organizational structure requires comparisons for other cases, which leads to an intriguing point in relation with a firm's boundary. Consider, particularly, that between the ex ante contracting and the partial, given that the simultaneous contracting is not optimal. By Proposition 2, an informed mechanism designer has an incentive to acquire the information as long as $c<V_{s}-\bar{U}-V_{p}$. Hence, under the condition, if the principal sells the project, the manager is willing to observe the information. Despite the willingness on the part of the manager, the optimality of the partial contracting means that the principal shall not confer such an opportunity, because to do so, the sale price is too expensive; rather take the partial contracting only with the agent. That is, the manager's chance for the information acquisition is obstructed by the principal.

Overall, the dominance of an organizational structure relies on the interplay between the extent to which the effective limited liability constrains the principal's payoff and the magnitude of information cost. ${ }^{36}$ Specifically, the interplay is provided through the threshold $k(\Delta U)$ that depends on $\Delta U$ given the limited liability.

Theorem 3 Suppose the effective limited liability $\Delta U>0$. If $k(\Delta U)<V_{\min }-B-V_{p}$, the optimal mechanism is

\footnotetext{
${ }^{36}$ Th equal sign is omitted in the Theorem since it simply means indifferent relationship, and its proof also contains conditions under which each case exists, in addition to its optimality.
} 


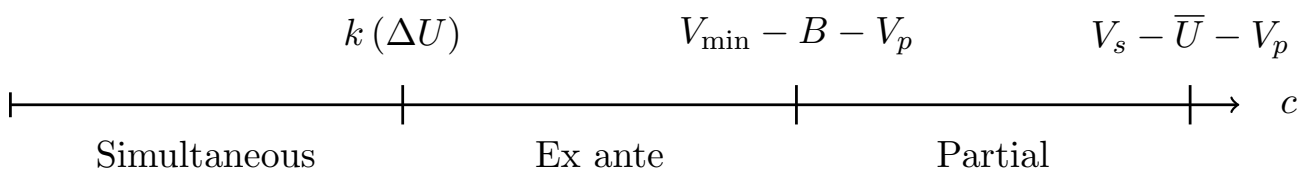

Figure 4: Three optimal mechanisms

(i) the simultaneous contracting if $c<k(\Delta U)$;

(ii) the ex ante contracting if $k(\Delta U)<c<V_{\min }-B-V_{p}$;

(iii) the partial contracting if $V_{\min }-B-V_{p}<c<V_{s}-\bar{U}-V_{p}$.

If $k(\Delta U)>V_{\min }-B-V_{p}$, for $\widehat{c} \in\left(V_{\min }-B-V_{p}, k(\Delta U)\right)$, the optimal mechanism is

(i) the simultaneous contracting if $c<\widehat{c}$;

(ii) the partial contracting if $\widehat{c}<c<V_{s}-\bar{U}-V_{p}$.

If the cost is in the low range, relative to $k(\Delta U)$, the optimal mechanism is to include the manager inside the firm. An additional and interesting implication is that the information acquisition can suggest when to sell a project by "outsourcing" it to an informed expert; if the cost is in the middle range. If it is high, the optimal mechanism is not to resort to a third party at all. The characterization with the three structures can be reduced to two if the limited liability can affect the principal severely for selling such that $k(\Delta U)>V_{\min }-B-V_{p}$ : It is optimal for the principal to "bypass" the ex ante contracting and rather take the partial contracting.

Now, suppose $\Delta U=0$ or $V_{s}-\bar{U} \leq V_{\min }-B$ so that limited liability is not effective at all. Then, the principal can always achieve the second-best payoff. Yet, the simultaneous contracting is not feasible if the information cost is high, which means that, in contrast to the second best's information acquisition in Proposition 2, if $k(0)<c<V_{s}-\bar{U}-V_{p}$, the principal still achieves the second best not through information acquisition but only by the selling.

Corollary 1 Suppose $\Delta U=0$, the optimal mechanism is

(i) the simultaneous and the ex ante contracting if $c<k(0)$;

(ii) the ex ante contracting if $k(0)<c<V_{s}-\bar{U}-V_{p}$.

\subsection{Boundary of the firm application}

We consider the comparison only between the simultaneous contracting and the ex-ante contracting to answer the question raised by Holmström and Roberts (1999). In Japan (especially, in the auto industry), despite the potential hold-up problem, auto makers largely depend on external suppliers for designing parts and manufacturing other components. They provide a convincing explanation on how it still works in a relationship of repeated transactions and information sharing, but not on what may originate such a system. The difference in information cost or difference in the maximum rent with three types of labor market can be suggested as the origin in this paper.

One immediate answer can be obtained if, for an identical maximum rent $\Delta U$ to the manager, one labor market's information cost $c^{\prime}$ is higher than the other's $c$ such that $c<k(\Delta U)<c^{\prime}$; that 
is, if this difference in the cost accounts for the difference in labor market between the U.S. and Japan. On the other hand, if the cost is identical for two regimes, we can consider different types of labor market with the principal's operating cost $a>0$, which can yield three different maximum rents as in Table $2, \Delta U^{I I}<\Delta U^{I}<\Delta U^{I I I}$ and so $k\left(\Delta U^{I I}\right)<k\left(\Delta U^{I}\right)<k\left(\Delta U^{I I I}\right) .{ }^{37}$

\begin{tabular}{l|l|l|l} 
& Large firm & Small firm & Maximum rent to M \\
\hline Labor market I & Flexible & Flexible & $\Delta U^{I}=V_{s}^{f}-\bar{U}-\left(V_{\min }-B\right)$ \\
\hline Labor market II & Not flexible & Flexible & $\Delta U^{I I}=V_{s}^{n}-\bar{U}-\left(V_{\min }-B\right)$ \\
\hline Labor market III & Not flexible & Not flexible & $\Delta U^{I I I}=V_{s}^{n}-\bar{U}-\left(V_{\min }-a-B\right)$
\end{tabular}

Table 2: Three types of labor market

Hence, if, in two different countries, $c$ is distributed among firms with the same distribution, a greater number of integrated firms will be found in the overall flexible labor market (Labor market I) versus the only flexible market for small firms (Labor market II), as in the U.S. versus Japan. ${ }^{38}$ Furthermore, this result predicts that the overall non-flexible labor market will have even more integrated firms, compared with the overall flexible labor market, due to the equally inflexible labor contracts with small firms.

\section{Concluding Remarks}

This paper studies different mechanism design problems in which a principal faces an agent whose output consists of two tasks: quantity and non-contractible quality. By comparing the simultaneous contracting with the ex ante contracting, we show that the former dominates the latter if the information cost is low. This dominance, combined with the maximum admissible cost and limited liability, results in three optimal mechanisms - simultaneous contracting, ex ante contracting, and partial contracting - and their respective indirect organizational structures such as the manager inside the firm, out-sourcing, and no quality assessment. The nature of contractual incompleteness between the principal and the agent of this model suggests a foundation of incomplete contract, non-contractible quality coupled with information cost and limited liability, and thus demands a different solution: the first-order alignment with the manager. This result has new implications for

\footnotetext{
${ }^{37}$ One modelling choice to endogenously derive such differences is to suppose that the principal incurs an operating cost $a>0$, including the minimum wage, for each worker with $v(\omega, 0)=0$, and to consider two different types of labor contract in terms of how flexible a contract with the worker is such that$$
\text { Flexible contract }\left\{\begin{array} { l l } 
{ v ( \omega , q ) - t - a } & { \text { if } q > 0 , } \\
{ v ( \omega , 0 ) } & { \text { if } q = 0 . }
\end{array} \text { Non flexible contract } \left\{\begin{array}{ll}
v(\omega, q)-t-a & \text { if } q>0, \\
v(\omega, 0)-a & \text { if } q=0 .
\end{array}\right.\right.
$$

where with the flexible contract, the principal can make the worker not produce whenever it is profitable to do so, so the optimal threshold cost type is given as an implicit function $\widehat{\theta}(\omega)$ satisfying $V(\widehat{\theta}(\omega), \omega)-a=0$. On the other hand, to "offset" the non-flexible contract, we assume that the principal can devise a test to screen a work quality level lower than $r$.

${ }^{38}$ Japan's labor market segmentation between large and small firms is well documented by Rebick (1993). On the other hand, according to Clark, Chew and Fujimoto (1987), Europe is between the U.S. and Japan in terms of relying on the number of parts suppliers (see pp.741-742), but it would be far-fetched to call it an overall non-flexible labor market as in Europe a relatively lucrative unemployment package plays a critical role (see Nickel (1997)).
} 
the theory of the firm to explain the origin of a firm's decentralized system, as opposed to what the standard mechanism theory offers.

The results of the present paper can be extended in several directions. The principal can implement the optimal contract in the form of delegation to the manager by restricting a feasible set of contracts that the manager chooses for the agent and offering the agent a take-it-or-leave-it decision. Suppose a pre-investment stage where the agent makes an investment. Then, for a positive investment cost, the partial contracting mechanism lowers not only the payoffs to the principal and the agent but also the incentive to make such investment. This decreases the principal's payoff further, compared with the second-best payoff. Finally, the analysis for a single agent can be extended to multiple agents if team production between them has no complementarity, by replicating the analysis for one agent to many agents. ${ }^{39}$

The model can also be developed further for new approaches. If the quality is not a noncontractible type but can be elicited, then the partial contracting's payoff will increase with the truthful report, even without the manager, so the structure including a third party will less likely be optimal. Yet, the exact details in the approach require broad and careful study - e.g. quality can be a type that can be elicited or an action with a different type specification - which is beyond our scope, but such quality often exists in the form of a worker's intrinsic type. ${ }^{40}$ The agent can also be protected by limited liability, as, in particular, in reality, but the main purpose of the paper is to show whether including the manager inside the firm is optimal, for which the manager's limited liability plays a role, whereas the agent's limited liability will equally affect either case. The focus of this paper is on how the principal can extract relevant information from a third party, the manager, so we restrict the analysis to a coalition-free environment, leaving a full characterization of collusion-proof mechanisms with non-contractible quality for future research.

\section{Appendix A Proofs}

Proof of Proposition 1. Step 1. We show that the incentive compatibility (3) is equivalently rewritten such that for any pair $(\theta, \omega),\left(\theta^{\prime}, \omega^{\prime}\right) \in \Theta \times \Omega$,

$$
\begin{aligned}
& t(\theta, \omega)-q(\theta, \omega) \theta \geq t\left(\theta^{\prime}, \omega\right)-q\left(\theta^{\prime}, \omega\right) \theta, \\
& t(\theta, \omega)-q(\theta, \omega) \theta=t\left(\theta, \omega^{\prime}\right)-q\left(\theta, \omega^{\prime}\right) \theta .
\end{aligned}
$$

First, show (3) $\Rightarrow(32)$-(33). If we fix $\omega \in \Omega$ or fix $\theta \in \Theta$, it is immediate that the incentive compatibility (3) implies the incentive compatibility (32)-(33). In particular, for the latter, i.e., for a fixed $\theta$, suppose that $\omega$ is the agent's (true) quality. Then, his incentive compatibility is satisfied

\footnotetext{
${ }^{39}$ In the case of a single indivisible good - such as a single unit auction - even with interactions between players, a similar analysis can be extended as in Yoo (2017). Technically, in the single-agent case, finding the optimal contract for the manager amounts to deriving an ordinary differential equation, which is still applicable even with multiple agents if there is no complementarity. If the type of their joint production involves complementarity, however, the analysis will not be tractable, since finding the optimal contract requires solving a system of partial differential equations, especially given the nonlinear nature of the problem.

${ }^{40}$ For evidence that such a trait exists as a type, i.e., quality in the present paper, see Burks et al (2015) and Pallais and Sands (2016).
} 
if the agent obtains a higher payoff from reporting it truthfully compared with the payoff from misreporting it such that

$$
t(\theta, \omega)-q(\theta, \omega) \theta \geq t\left(\theta, \omega^{\prime}\right)-q\left(\theta, \omega^{\prime}\right) .
$$

On the other hand, suppose that $\omega^{\prime}$ is the agent's quality. Then, his incentive compatibility is satisfied if

$$
t\left(\theta, \omega^{\prime}\right)-q\left(\theta, \omega^{\prime}\right) \theta \geq t(\theta, \omega)-q(\theta, \omega) .
$$

Together, the equality in (33) is derived. ${ }^{41}$ In deriving the equality, we use the fact that the true quality does not affect the agent's payoff, so it does not appear in it; in other words, any $\omega$ or $\omega^{\prime}$ appearing in the agent's payoff is his report (again, it is not the agent's value but the principal's). Further, (33) implies that for any mechanism inducing the agent to report $\omega$ truthfully for a fixed $\theta$, the agent must have an identical payoff.

Now, show $(3) \Leftarrow(32)-(33)$. By combining (32) with (33), it is immediate that the latter implies the former.

Step 2. From the incentive compatibility (32)-(33), a direct mechanism $(q, t)$ is incentive compatible if and only if (i) $q$ is decreasing in $\theta$; (ii) for any $\theta \in \Theta$,

$$
t(\theta, \omega)=q(\theta, \omega) \theta+(t(\bar{\theta}, \omega)-q(\bar{\theta}, \omega) \bar{\theta})+\int_{\theta}^{\bar{\theta}} q(x, \omega) d x
$$

and (iii) for each $\omega \neq \omega^{\prime} \in \Omega$,

$$
t(\theta, \omega)-q(\theta, \omega) \theta=t\left(\theta, \omega^{\prime}\right)-q\left(\theta, \omega^{\prime}\right) \theta .
$$

Consider $(\theta, \omega),\left(\theta, \omega^{\prime}\right) \in \Theta \times \Omega$ with $\omega^{\prime} \neq \omega$. Define $D(\theta)$ such that

$$
D(\theta) \equiv t(\bar{\theta}, \omega)-q(\bar{\theta}, \omega) \bar{\theta}+\int_{\theta}^{\bar{\theta}} q(x, \omega) d x-\left[t\left(\bar{\theta}, \omega^{\prime}\right)-q\left(\bar{\theta}, \omega^{\prime}\right) \bar{\theta}+\int_{\theta}^{\bar{\theta}} q\left(x, \omega^{\prime}\right) d x\right] .
$$

Then, from (34), we have

$$
\begin{aligned}
t(\theta, \omega)-q(\theta, \omega) \theta & =(t(\bar{\theta}, \omega)-q(\bar{\theta}, \omega) \bar{\theta})+\int_{\theta}^{\bar{\theta}} q(x, \omega) d x \\
t\left(\theta, \omega^{\prime}\right)-q\left(\theta, \omega^{\prime}\right) \theta & =\left(t\left(\bar{\theta}, \omega^{\prime}\right)-q\left(\bar{\theta}, \omega^{\prime}\right) \bar{\theta}\right)+\int_{\theta}^{\bar{\theta}} q\left(x, \omega^{\prime}\right) d x .
\end{aligned}
$$

Then, by (33), $D(\theta)=0$ for all $\theta \in \Theta$. It follows from the mean value theorem and the fundamental theorem of calculus that $D^{\prime}(\theta)=0$ for almost all $\theta \in \Theta$. Hence, for each $\omega \neq \omega^{\prime} \in \Omega, q(\theta, \omega)=$ $q\left(\theta, \omega^{\prime}\right)$ and $t(\theta, \omega)=t\left(\theta, \omega^{\prime}\right)$ for almost all $\theta \in \Theta$.

Proof of Proposition 2. To make the analysis self-contained, with a slight abuse of notations, we denote by $\widehat{G}(\theta, \omega)$ the joint CDF of $\theta$ and $\omega$ and by $G_{\theta}(\theta)$ the marginal CDF of $\theta$.

Part 1 Find $V_{p}$ for (4). With $\left(q_{p}, t_{p}\right), \Gamma^{p}$ is incentive compatible if for each $\theta, \theta^{\prime} \in \Theta, t_{p}(\theta)-$ $q_{p}(\theta) \theta \geq t_{p}\left(\theta^{\prime}\right)-q_{p}\left(\theta^{\prime}\right) \theta$, and it is individually rational if for each $\theta \in \Theta, t_{p}(\theta)-q_{p}(\theta) \theta \geq 0$.

\footnotetext{
${ }^{41}$ One need (32) as well as (33) for (3). That is, the agent's incentive compatibility must also take care of misreporting $\theta$. For instance, consider a contract $q(\theta, \omega)=\omega / \theta$ and $t(\theta, \omega)=\omega$, but if the agent misreports $\theta^{\prime}<\theta$, then the incentive compatibility is violated: $t(\theta, \omega)-q(\theta, \omega) \theta=0<\omega-\left(\theta^{\prime} / \theta\right) \omega$.
} 
Under the principal's payoff maximization, by a standard analysis, an incentive compatible \& individually rational $\Gamma^{p}$ yields $t_{p}(\theta)=q_{p}(\theta) \theta+\int_{\theta}^{\bar{\theta}} q_{p}(x) d x$, and thus the principal's payoff in (4). Since $\widehat{G}(\theta, \omega)=F(\theta \mid \omega) G(\omega)$, this is equivalent to:

$$
\begin{aligned}
& \int_{\Theta \times \Omega}\left[v\left(\omega, q_{p}(\theta)\right)-q_{p}(\theta) \theta-\int_{\theta}^{\bar{\theta}} q_{p}(x) d x\right] d \widehat{G}(\theta, \omega) \\
& =\int_{\Theta}\left[\mathbb{E}_{\omega}\left[v\left(\omega, q_{p}(\theta)\right)\right]-q_{p}(\theta) \theta-\int_{\theta}^{\bar{\theta}} q_{p}(x) d x\right] d G_{\theta}(\theta) \\
& =\int_{\Theta}\left[\mathbb{E}_{\omega}\left[v\left(\omega, q_{p}(\theta)\right)\right]-q_{p}(\theta)\left(\theta+\frac{G_{\theta}(\theta)}{g_{\theta}(\theta)}\right)\right] d G_{\theta}(\theta) .
\end{aligned}
$$

The first equality follows since $v$ is the only term that depends on $\omega$ inside the integral, where $\mathbb{E}_{\omega}\left[v\left(\omega, q_{p}(\theta)\right)\right]$ is the expected value of $v\left(\omega, q_{p}(\theta)\right)$ given $\omega$ that the principal obtains. The second equality follows from the standard procedure by changing the order of integration. Then, the principal chooses $q_{p}$ to maximize the term inside the integral, which yields $V_{p}$.

Part 2 Find $V_{s}$ for (7). For the benchmark, its direct mechanism yields the principal's payoff such as

$$
\int_{\Omega} \int_{\Theta}\left[v\left(\omega, q_{s}(\theta, \omega)\right)-q_{s}(\theta, \omega) \theta-\int_{\theta}^{\bar{\theta}} q_{s}(x, \omega) d x\right] f(\theta \mid \omega) d \theta d G(\omega),
$$

which, by changing the order of integration, is rewritten as (7). The principal chooses $q_{s}$ to maximize the term inside the integral, and the solution yields $V_{s}$.

Part 3 Compare them. Since $\widehat{G}(\theta, \omega)=F(\theta \mid \omega) G(\omega)$,

$$
\begin{aligned}
& \int_{\Theta \times \Omega}\left[v\left(\omega, q_{p}(\theta)\right)-q_{p}(\theta) \theta-\int_{\theta}^{\bar{\theta}} q_{p}(x) d x\right] d \widehat{G}(\theta, \omega) \\
= & \int_{\Omega} \int_{\Theta}\left[v\left(\omega, q_{p}(\theta)\right)-q_{p}(\theta) \theta-\int_{\theta}^{\bar{\theta}} q_{p}(x) d x\right] f(\theta \mid \omega) d \theta d G(\omega) \\
= & \int_{\Omega} \int_{\Theta}\left[v\left(\omega, q_{p}(\theta)\right)-q_{p}(\theta) \phi(\theta, \omega)\right] f(\theta \mid \omega) d \theta d G(\omega),
\end{aligned}
$$

where we apply changing the order of integration with respect to $f(\theta \mid \omega)$ this time. It is clear that for all $(\theta, \omega), v\left(\omega, q_{s}(\theta, \omega)\right)-q_{s}(\theta, \omega) \phi(\theta, \omega) \geq v\left(\omega, q_{p}(\theta)\right)-q_{p}(\theta) \phi(\theta, \omega)$. Furthermore, the optimal interior $q_{s}$ in (8) is strictly increasing in $\omega$, whereas $q_{p}$ is a constant over all $\omega$, so by comparing the payoff $V_{s}$ from (7) with $V_{p}$, we have the strict inequality such that

$$
\begin{aligned}
V_{s} & =\int_{\Omega} \int_{\Theta}\left[v\left(\omega, q_{s}(\theta, \omega)\right)-q_{s}(\theta, \omega) \phi(\theta, \omega)\right] f(\theta \mid \omega) d \theta d G(\omega) \\
& >\int_{\Omega} \int_{\Theta}\left[v\left(\omega, q_{p}(\theta)\right)-q_{p}(\theta) \phi(\theta, \omega)\right] f(\theta \mid \omega) d \theta d G(\omega)=V_{p} .
\end{aligned}
$$

where the last equality follows from (36).

Proof of Proposition 3. By the envelope theorem, for $q_{s}(\theta, \omega)>0$,

$$
V_{\theta}(\theta, \omega)=-q_{s}(\theta, \omega) \phi_{\theta}(\theta, \omega)<0 ; V_{\omega}(\theta, \omega)=v_{\omega}\left(\omega, q_{s}(\theta, \omega)\right)-q_{s}(\theta, \omega) \phi_{\omega}(\theta, \omega)>0,
$$


where the strict inequalities follow from our assumptions. Then, it is clear that $V_{s}=\mathbb{E}[V(\theta, \omega)]>$ $\min _{(\theta, \omega)} V(\theta, \omega)$. Hence, the principal's payoff from the optimal ex ante mechanism satisfying the individual rationality including the limited liability is given as

$$
\alpha= \begin{cases}V_{s}-\bar{U}-c & \text { if } V_{s}-\bar{U} \leq \min _{(\theta, \omega)} V(\theta, \omega)-B \\ \min _{(\theta, \omega)} V(\theta, \omega)-B-c & \text { if } V_{s}-\bar{U}>\min _{(\theta, \omega)} V(\theta, \omega)-B .\end{cases}
$$

If $V_{p} \geq \min _{(\theta, \omega)} V(\theta, \omega)-B$, we have $\min _{(\theta, \omega)} V(\theta, \omega)-B<V_{s}-\bar{U}$ for all $\bar{U}<V_{s}-V_{p}$, since $-V_{p} \leq-\min _{(\theta, \omega)} V(\theta, \omega)+B$, so $V_{s}-V_{p} \leq V_{s}-\min _{(\theta, \omega)} V(\theta, \omega)+B$. On the other hand, if $V_{p}<\min _{(\theta, \omega)} V(\theta, \omega)-B$, then $\min _{(\theta, \omega)} V(\theta, \omega)-B<V_{s}-\bar{U}$ for some $\bar{U}<V_{s}-V_{p}$.

Proof of Lemma 1. We first show the following claim that will be used in Step 1 repeatedly. Note that $\operatorname{Pr}\left(q_{s}\left(\theta, \omega^{\prime}\right)>q_{i} \mid \omega\right)>0$ for all $\omega, \omega^{\prime} \in \Omega$ is equivalent to $q_{i}<q_{s}(\underline{\theta}, \underline{\omega})$, and we use the claim later for the negation of $q_{i}<q_{s}(\underline{\theta}, \underline{\omega})$, i.e. $q_{i} \geq q_{s}(\underline{\theta}, \underline{\omega})$.

Claim. If $q_{i} \geq q_{s}(\underline{\theta}, \underline{\omega})$, for each $\omega, \omega^{\prime \prime} \in \Omega$,

$$
\operatorname{Pr}\left(q_{s}\left(\theta, \omega^{\prime}\right)>q_{i} \mid \omega\right)>0(=0) \Leftrightarrow \operatorname{Pr}\left(q_{s}\left(\theta, \omega^{\prime}\right)>q_{i} \mid \omega^{\prime \prime}\right)>0(=0) .
$$

Proof. Since $q_{s}$ is a strictly increasing function of $\omega$, for $q_{i} \geq q_{s}(\underline{\theta}, \underline{\omega})$, there exists a cut-off value $\widetilde{\omega}\left(q_{i}\right) \geq \underline{\omega}$ satisfying $q_{s}\left(\underline{\theta}, \widetilde{\omega}\left(q_{i}\right)\right)=q_{i}$ such that $q_{s}\left(\underline{\theta}, \omega^{\prime}\right) \leq q_{i}$ if $\omega^{\prime} \leq \widetilde{\omega}\left(q_{i}\right)$, whereas $q_{s}\left(\underline{\theta}, \omega^{\prime}\right)>$ $q_{i}$ if $\omega^{\prime}>\widetilde{\omega}\left(q_{i}\right)$. Then,

$$
\left\{\begin{array}{ll}
\operatorname{Pr}\left(q_{s}\left(\theta, \omega^{\prime}\right)>q_{i} \mid \omega\right)=0 & \text { if } \omega^{\prime} \leq \widetilde{\omega}\left(q_{i}\right), \\
\operatorname{Pr}\left(q_{s}\left(\theta, \omega^{\prime}\right)>q_{i} \mid \omega\right)>0 & \text { if } \omega^{\prime}>\widetilde{\omega}\left(q_{i}\right),
\end{array} \text { for all } \omega \in \Omega\right.
$$

which yields the claim. - That is, whether the probability is positive or not does not depend on the true quality.

Now, the two results of the lemma can be shown with the following three steps.

Step 1. Show that $s_{i}\left(\omega^{\prime}\right) \operatorname{Pr}\left(q_{s}\left(\theta, \omega^{\prime}\right)>q_{i} \mid \omega\right) \neq 0$ for all $\omega, \omega^{\prime} \in \Omega$. Suppose, on the contrary, that there exist $\omega, \omega^{\prime} \in \Omega$ such that $s_{i}\left(\omega^{\prime}\right) \operatorname{Pr}\left(q_{s}\left(\theta, \omega^{\prime}\right)>q_{i} \mid \omega\right)=0$, which is equivalent to $q_{i} \geq q_{s}(\underline{\theta}, \underline{\omega})$. First, show that if $s_{i}\left(\omega^{\prime}\right) \operatorname{Pr}\left(q_{s}\left(\theta, \omega^{\prime}\right)>q_{i} \mid \omega\right)=0$, then

$$
s_{i}\left(\omega^{\prime}\right) \operatorname{Pr}\left(q_{s}\left(\theta, \omega^{\prime}\right)>q_{i} \mid \omega^{\prime}\right)=0 \text {, that is, conditional on } \omega^{\prime} .
$$

If $s_{i}\left(\omega^{\prime}\right)=0$, the above trivially holds; now, if $s_{i}\left(\omega^{\prime}\right) \neq 0$, by $(37), \operatorname{Pr}\left(q_{s}\left(\theta, \omega^{\prime}\right)>q_{i} \mid \omega\right)=0$ implies $\operatorname{Pr}\left(q_{s}\left(\theta, \omega^{\prime}\right)>q_{i} \mid \omega^{\prime}\right)=0$. On the other hand, for target $i$ such that there exists $\omega, \omega^{\prime \prime} \in \Omega$ such that $s_{i}\left(\omega^{\prime \prime}\right) \operatorname{Pr}\left(q_{s}\left(\theta, \omega^{\prime \prime}\right)>q_{i} \mid \omega\right) \neq 0$, which implies $s_{i}\left(\omega^{\prime \prime}\right) \neq 0$ and $\operatorname{Pr}\left(q_{s}\left(\theta, \omega^{\prime \prime}\right)>q_{i} \mid \omega\right)>0$. By $(37)$, $\operatorname{Pr}\left(q_{s}\left(\theta, \omega^{\prime \prime}\right)>q_{i} \mid \omega\right)>0$ implies $\operatorname{Pr}\left(q_{s}\left(\theta, \omega^{\prime \prime}\right)>q_{i} \mid \omega^{\prime}\right)>0$ as well as $\operatorname{Pr}\left(q_{s}\left(\theta, \omega^{\prime \prime}\right)>q_{i} \mid \omega^{\prime \prime}\right)>0$. Hence,

$$
s_{i}\left(\omega^{\prime \prime}\right) \operatorname{Pr}\left(q_{s}\left(\theta, \omega^{\prime \prime}\right)>q_{i} \mid \omega^{\prime}\right) \neq 0 .
$$

For $s_{i}\left(\omega^{\prime \prime}\right)>0, s_{i}\left(\omega^{\prime}\right) \operatorname{Pr}\left(q_{s}\left(\theta, \omega^{\prime}\right)>q_{i} \mid \omega^{\prime}\right)=0<s_{i}\left(\omega^{\prime \prime}\right) \operatorname{Pr}\left(q_{s}\left(\theta, \omega^{\prime \prime}\right)>q_{i} \mid \omega^{\prime}\right)$. For $s_{i}\left(\omega^{\prime \prime}\right)<$ $0, s_{i}\left(\omega^{\prime \prime}\right) \operatorname{Pr}\left(q_{s}\left(\theta, \omega^{\prime \prime}\right)>q_{i} \mid \omega^{\prime \prime}\right)<0=s_{i}\left(\omega^{\prime}\right) \operatorname{Pr}\left(q_{s}\left(\theta, \omega^{\prime}\right)>q_{i} \mid \omega^{\prime \prime}\right)$, since if $s_{i}\left(\omega^{\prime}\right)=0$, $s_{i}\left(\omega^{\prime}\right) \operatorname{Pr}\left(q_{s}\left(\theta, \omega^{\prime}\right)>q_{i} \mid \omega^{\prime \prime}\right)=0$; if not, by $(37), \operatorname{Pr}\left(q_{s}\left(\theta, \omega^{\prime}\right)>q_{i} \mid \omega^{\prime}\right)=0$ implies $\operatorname{Pr}\left(q_{s}\left(\theta, \omega^{\prime}\right)>\right.$ $\left.q_{i} \mid \omega^{\prime \prime}\right)=0$. This contradicts IC.

Step 2. From Step 1, $u\left(\omega^{\prime}, \omega, q_{i}\right)=s_{i}\left(\omega^{\prime}\right) F\left(p\left(\omega^{\prime}, q_{i}\right) \mid \omega\right)$ and $s_{i}\left(\omega^{\prime}\right) F\left(p\left(\omega^{\prime}, q_{i}\right) \mid \omega\right) \neq 0$ for all $\omega, \omega^{\prime} \in \Omega$, which also implies $s_{i}(\omega) \neq 0$ for all $\omega \in \Omega$. We divide this step into two parts. 
Part 1. $s_{i}(\omega)>0$ for all $\omega \in \Omega$. (a) We first show that $s_{i}(\omega)$ is a strictly decreasing function of $\omega$. Any incentive compatible $s_{i}(\omega)$ satisfies that for any $\omega^{\prime}>\omega$,

$$
s_{i}(\omega) F\left(p\left(\omega, q_{i}\right) \mid \omega\right) \geq s_{i}\left(\omega^{\prime}\right) F\left(p\left(\omega^{\prime}, q_{i}\right) \mid \omega\right) .
$$

For $q_{i} \geq 0$ satisfying Step 1 , we have $q_{s}\left(p\left(\omega, q_{i}\right), \omega\right)=q_{i}$, which guarantees that $p\left(\omega, q_{i}\right)$ is a strictly increasing function of $\omega$. Suppose, on the contrary, that $s_{i}\left(\omega^{\prime}\right) \geq s_{i}(\omega)$ for $\omega^{\prime}>\omega$. Then, $s_{i}(\omega) F\left(p\left(\omega, q_{i}\right) \mid \omega\right)<s_{i}\left(\omega^{\prime}\right) F\left(p\left(\omega^{\prime}, q_{i}\right) \mid \omega\right)$, which contradicts IC.

(b) $s_{i}(\omega)$ is continuous on $[\underline{\omega}, \bar{\omega}]$. First, note that the separable incentive compatibility IC for $s_{i}(\omega)$ satisfies that for each $\omega, \omega^{\prime} \in \Omega$,

$$
\left\{\begin{array}{c}
s_{i}\left(\omega^{\prime}\right) F\left(p\left(\omega^{\prime}, q_{i}\right) \mid \omega^{\prime}\right) \geq s_{i}(\omega) F\left(p\left(\omega, q_{i}\right) \mid \omega^{\prime}\right) \\
s_{i}(\omega) F\left(p\left(\omega, q_{i}\right) \mid \omega\right) \geq s_{i}\left(\omega^{\prime}\right) F\left(p\left(\omega^{\prime}, q_{i}\right) \mid \omega\right)
\end{array}\right.
$$

Now, we examine an interior point and two corner points separately. Show that $s_{i}(\omega)$ is continuous at $\underline{\omega}$. For each $\omega^{\prime}>\omega=\underline{\omega}, \mathrm{IC}$ in (38) is satisfied. Suppose $s_{i}(\omega)$ is not continuous at $\underline{\omega}$. Then by (a) of Part 1, we have $s_{i}(\underline{\omega})>\lim _{\omega \rightarrow \underline{\omega}}+s_{i}(\omega)$. Let $\omega^{\prime} \rightarrow \underline{\omega}$. In the limit, the first IC becomes

$$
\lim _{\omega^{\prime} \rightarrow \underline{\omega}+} s_{i}\left(\omega^{\prime}\right) F\left(p\left(\underline{\omega}, q_{i}\right) \mid \underline{\omega}\right) \geq s_{i}(\underline{\omega}) F\left(p\left(\underline{\omega}, q_{i}\right) \mid \underline{\omega}\right) \Leftrightarrow \lim _{\omega^{\prime} \rightarrow \underline{\omega}+} s_{i}\left(\omega^{\prime}\right) \geq s_{i}(\underline{\omega})
$$

which is a contradiction. Show that $s_{i}(\omega)$ is continuous at $\bar{\omega}$. For each $\omega^{\prime}<\omega=\bar{\omega}$, the IC in (38) is satisfied. Suppose $s_{i}(\omega)$ is not continuous at $\bar{\omega}$. Then by (a) of Part 1 , we have $\lim _{\omega \rightarrow \bar{\omega}}-s_{i}(\omega)>$ $s_{i}(\bar{\omega})$. Let $\omega^{\prime} \rightarrow \underline{\omega}$. In the limit, the second IC becomes

$$
s_{i}(\bar{\omega}) F\left(p\left(\bar{\omega}, q_{i}\right) \mid \bar{\omega}\right) \geq \lim _{\omega \rightarrow \bar{\omega}-} s_{i}(\omega) F\left(p\left(\bar{\omega}, q_{i}\right) \mid \bar{\omega}\right) \Leftrightarrow s_{i}(\bar{\omega}) \geq \lim _{\omega \rightarrow \bar{\omega}-} s_{i}(\omega)
$$

and a contradiction. Show that $s_{i}(\omega)$ is continuous at $\omega \in(\underline{\omega}, \bar{\omega})$. For each $\omega \neq \omega^{\prime} \in(\underline{\omega}, \bar{\omega})$, the IC in (38) is satisfied. We let $\omega^{\prime} \rightarrow \omega$. Then,

$$
\begin{aligned}
& \left\{\begin{array}{l}
\lim _{\omega^{\prime} \rightarrow \omega} s_{i}\left(\omega^{\prime}\right) F\left(p\left(\omega, q_{i}\right) \mid \omega\right) \geq s_{i}(\omega) F\left(p\left(\omega, q_{i}\right) \mid \omega\right), \\
s_{i}(\omega) F\left(p\left(\omega, q_{i}\right) \mid \omega\right) \geq \lim _{\omega^{\prime} \rightarrow \omega} s_{i}\left(\omega^{\prime}\right) F\left(p\left(\omega, q_{i}\right) \mid \omega\right)
\end{array}\right. \\
\Leftrightarrow & \lim _{\omega^{\prime} \rightarrow \omega} s_{i}\left(\omega^{\prime}\right) \geq s_{i}(\omega) \geq \lim _{\omega^{\prime} \rightarrow \omega} s_{i}\left(\omega^{\prime}\right) .
\end{aligned}
$$

(c) $s_{i}(\omega)$ is a differentiable function of $\omega$. Consider IC in (38). For each $\omega \neq \omega^{\prime}$,

$$
\begin{aligned}
& \left\{\begin{array}{l}
s_{i}\left(\omega^{\prime}\right) F\left(p\left(\omega^{\prime}, q_{i}\right) \mid \omega^{\prime}\right) \geq s_{i}(\omega) F\left(p\left(\omega, q_{i}\right) \mid \omega^{\prime}\right), \\
s_{i}(\omega) F\left(p\left(\omega, q_{i}\right) \mid \omega\right) \geq s_{i}\left(\omega^{\prime}\right) F\left(p\left(\omega^{\prime}, q_{i}\right) \mid \omega\right) .
\end{array}\right. \\
\Leftrightarrow & \left\{\begin{array}{c}
s_{i}\left(\omega^{\prime}\right) F\left(p\left(\omega^{\prime}, q_{i}\right) \mid \omega^{\prime}\right)-s_{i}(\omega) F\left(p\left(\omega^{\prime}, q_{i}\right) \mid \omega^{\prime}\right) \geq s_{i}(\omega) F\left(p\left(\omega, q_{i}\right) \mid \omega^{\prime}\right)-s_{i}(\omega) F\left(p\left(\omega^{\prime}, q_{i}\right) \mid \omega^{\prime}\right), \\
s_{i}(\omega) F\left(p\left(\omega, q_{i}\right) \mid \omega\right)-s_{i}(\omega) F\left(p\left(\omega^{\prime}, q_{i}\right) \mid \omega\right) \geq s_{i}\left(\omega^{\prime}\right) F\left(p\left(\omega^{\prime}, q_{i}\right) \mid \omega\right)-s_{i}(\omega) F\left(p\left(\omega^{\prime}, q_{i}\right) \mid \omega\right),
\end{array}\right.
\end{aligned}
$$

which becomes

$$
\Leftrightarrow\left\{\begin{array}{l}
\frac{s_{i}\left(\omega^{\prime}\right)-s_{i}(\omega)}{\omega^{\prime}-\omega} F\left(p\left(\omega^{\prime}, q_{i}\right) \mid \omega^{\prime}\right) \geq-\frac{F\left(p\left(\omega, q_{i}\right) \mid \omega^{\prime}\right)-F\left(p\left(\omega^{\prime}, q_{i}\right) \mid \omega^{\prime}\right)}{\omega-\omega^{\prime}} s_{i}(\omega), \\
-\frac{F\left(p\left(\omega, q_{i}\right) \mid \omega\right)-F\left(p\left(\omega^{\prime}, q_{i}\right) \mid \omega\right)}{\omega-\omega^{\prime}} s_{i}(\omega) \geq \frac{s_{i}\left(\omega^{\prime}\right)-s_{i}(\omega)}{\omega^{\prime}-\omega} F\left(p\left(\omega^{\prime}, q_{i}\right) \mid \omega\right) .
\end{array}\right.
$$


In the limit, we have

$$
\begin{aligned}
{\left[\lim _{\omega^{\prime} \rightarrow \omega} \frac{s_{i}\left(\omega^{\prime}\right)-s_{i}(\omega)}{\omega^{\prime}-\omega}\right] F\left(p\left(\omega, q_{i}\right) \mid \omega\right) } & \geq-s_{i}(\omega) f\left(p\left(\omega, q_{i}\right) \mid \omega\right) p_{\omega}\left(\omega, q_{i}\right) \\
& \geq\left[\lim _{\omega^{\prime} \rightarrow \omega} \frac{s_{i}\left(\omega^{\prime}\right)-s_{i}(\omega)}{\omega^{\prime}-\omega}\right] F\left(p\left(\omega, q_{i}\right) \mid \omega\right) .
\end{aligned}
$$

Part 2. $s_{i}(\omega)<0$ for all $\omega \in \Omega$. We show that $s_{i}(\omega)$ is now strictly increasing on $\Omega$. Suppose, on the contrary, that $s_{i}\left(\omega^{\prime}\right) \leq s_{i}(\omega)$ for $\omega^{\prime}>\omega$. Then, given the negative $s_{i}(\omega)<0$ for all $\omega \in \Omega$, $s_{i}\left(\omega^{\prime}\right) F\left(p\left(\omega^{\prime}, q_{i}\right) \mid \omega^{\prime}\right)<s_{i}\left(\omega^{\prime}\right) F\left(p\left(\omega, q_{i}\right) \mid \omega^{\prime}\right) \leq s_{i}(\omega) F\left(p\left(\omega, q_{i}\right) \mid \omega^{\prime}\right)$, which contradicts IC. Then, using the same procedure as Part 1 , it can be shown that $s_{i}(\omega)$ is differentiable.

Step 3. If $s_{i}(\omega) \operatorname{Pr}\left(q_{s}(\theta, \omega)>q_{i} \mid \omega\right)<0$ for all $\omega \in \Omega$, show that IC is violated. For $\omega^{\prime}>\omega$ to satisfy the first order condition,

$$
\begin{aligned}
0 & =u_{\omega^{\prime}}\left(\omega^{\prime}, \omega^{\prime}, q_{i}\right) \\
& =s_{i}^{\prime}\left(\omega^{\prime}\right) f\left(p\left(\omega^{\prime}, q_{i}\right) \mid \omega^{\prime}\right)\left[\frac{s_{i}\left(\omega^{\prime}\right) p_{\omega}\left(\omega^{\prime}, q_{i}\right)}{s_{i}^{\prime}\left(\omega^{\prime}\right)}+\frac{F\left(p\left(\omega^{\prime}, q_{i}\right) \mid \omega^{\prime}\right)}{f\left(p\left(\omega^{\prime}, q_{i}\right) \mid \omega^{\prime}\right)}\right] \\
& <s_{i}^{\prime}\left(\omega^{\prime}\right) f\left(p\left(\omega^{\prime}, q_{i}\right) \mid \omega^{\prime}\right)\left[\frac{s_{i}\left(\omega^{\prime}\right) p_{\omega}\left(\omega^{\prime}, q_{i}\right)}{s_{i}^{\prime}\left(\omega^{\prime}\right)}+\frac{F\left(p\left(\omega^{\prime}, q_{i}\right) \mid \omega\right)}{f\left(p\left(\omega^{\prime}, q_{i}\right) \mid \omega\right)}\right],
\end{aligned}
$$

where the last inequality follows from $s_{i}\left(\omega^{\prime}\right)<0, s_{i}^{\prime}\left(\omega^{\prime}\right)>0$ from Part 2 of Step 2 and the reverse hazard rate dominance. Then this implies that $\frac{s_{i}\left(\omega^{\prime}\right) p_{\omega}\left(\omega^{\prime}, q_{i}\right)}{s_{i}^{\prime}\left(\omega^{\prime}\right)}+\frac{F\left(p\left(\omega^{\prime}, q_{i}\right) \mid \omega\right)}{f\left(p\left(\omega^{\prime}, q_{i}\right) \mid \omega\right)}>0$, and thus for $\omega^{\prime}>\omega$,

$$
u_{\omega^{\prime}}\left(\omega^{\prime}, \omega, q_{i}\right)=s_{i}^{\prime}\left(\omega^{\prime}\right) f\left(p\left(\omega^{\prime}, q_{i}\right) \mid \omega\right)\left[\frac{s_{i}\left(\omega^{\prime}\right) p_{\omega}\left(\omega^{\prime}, q_{i}\right)}{s_{i}^{\prime}\left(\omega^{\prime}\right)}+\frac{F\left(p\left(\omega^{\prime}, q_{i}\right) \mid \omega\right)}{f\left(p\left(\omega^{\prime}, q_{i}\right) \mid \omega\right)}\right]>0 .
$$

Hence, misreporting $\omega^{\prime}$ yields a higher payoff. Together, by Step 3 eliminating $s_{i}(\omega)<0$ for all $\omega \in \Omega$, Step 1 and 2 establish (i) and (ii).

Proof of Theorem 1. Part 1 Equivalence. For $(16) \Rightarrow(17)$, the omitted steps between (20) and $(21)$ are relegated to Appendix B.2. Show $(16) \Leftarrow(17)$. The manager chooses $\omega^{\prime}$ to maximize $u\left(\omega^{\prime}, \omega, q_{i}\right)=s_{i}\left(\omega^{\prime}\right) F\left(p\left(\omega^{\prime}, q_{i}\right) \mid \omega\right)$. By Lemma 1, the partial derivative of $u\left(\omega^{\prime}, \omega, q_{i}\right)$ with respect to $\omega^{\prime}$ yields

$$
\begin{aligned}
u_{\omega^{\prime}}\left(\omega^{\prime}, \omega, q_{i}\right) & =-s_{i}^{\prime}\left(\omega^{\prime}\right) f\left(p\left(\omega^{\prime}, q_{i}\right) \mid \omega\right)\left[-\frac{s_{i}\left(\omega^{\prime}\right) p_{\omega}\left(\omega^{\prime}, q_{i}\right)}{s_{i}^{\prime}\left(\omega^{\prime}\right)}-\frac{F\left(p\left(\omega^{\prime}, q_{i}\right) \mid \omega\right)}{f\left(p\left(\omega^{\prime}, q_{i}\right) \mid \omega\right)}\right] \\
& =-s_{i}^{\prime}\left(\omega^{\prime}\right) f\left(p\left(\omega^{\prime}, q_{i}\right) \mid \omega\right)\left[p\left(\omega^{\prime}, q_{i}\right)-\frac{s_{i}\left(\omega^{\prime}\right) p_{\omega}\left(\omega^{\prime}, q_{i}\right)}{s_{i}^{\prime}\left(\omega^{\prime}\right)}-\phi\left(p\left(\omega^{\prime}, q_{i}\right), \omega\right)\right],
\end{aligned}
$$

where $\phi\left(p\left(\omega^{\prime}, q_{i}\right), \omega\right)=p\left(\omega^{\prime}, q_{i}\right)+F\left(p\left(\omega^{\prime}, q_{i}\right) \mid \omega\right) / f\left(p\left(\omega^{\prime}, q_{i}\right) \mid \omega\right)$. If the principal chooses $s_{i}(\omega)$ such that for each $\omega \in \Omega$ and $q \geq 0$,

$$
p\left(\omega, q_{i}\right)-\frac{s_{i}(\omega) p_{\omega}\left(\omega, q_{i}\right)}{s_{i}^{\prime}(\omega)}=v_{q}\left(\omega, q_{i}\right)
$$

the condition in (39) can be rewritten as $u_{\omega^{\prime}}\left(\omega^{\prime}, \omega, q_{i}\right)=-s_{i}^{\prime}\left(\omega^{\prime}\right) f\left(p\left(\omega^{\prime}, q_{i}\right) \mid \omega\right)\left[v_{q}\left(\omega^{\prime}, q_{i}\right)-\phi\left(p\left(\omega^{\prime}, q_{i}\right), \omega\right)\right]$. Since for $q_{i} \geq 0$ satisfying Lemma $1, q_{s}\left(p\left(\omega, q_{i}\right), \omega\right)=q_{i}$, the optimality condition of the second 
best (8) implies that for each $\omega \in \Omega$, the first-order condition for the manager's truthful report is satisfied such as $u_{\omega^{\prime}}\left(\omega, \omega, q_{i}\right)=0$. In other words,

$$
v_{q}\left(\omega, q_{i}\right)-\phi\left(p\left(\omega, q_{i}\right), \omega\right)=0 \Leftrightarrow v_{q}\left(\omega, q_{s}(\theta, \omega)\right)-\phi(\theta, \omega)=0 .
$$

Since for any $\omega^{\prime}>\omega$, the first order condition is also satisfied such as $u_{\omega^{\prime}}\left(\omega^{\prime}, \omega^{\prime}, q_{i}\right)=0$, we have

$$
\begin{aligned}
0 & =u_{\omega^{\prime}}\left(\omega^{\prime}, \omega^{\prime}, q_{i}\right) \\
& =-s_{i}^{\prime}\left(\omega^{\prime}\right) f\left(p\left(\omega^{\prime}, q_{i}\right) \mid \omega^{\prime}\right)\left[v_{q}\left(\omega^{\prime}, q_{i}\right)-\phi\left(p\left(\omega^{\prime}, q_{i}\right), \omega^{\prime}\right)\right] \\
& >-s_{i}^{\prime}\left(\omega^{\prime}\right) f\left(p\left(\omega^{\prime}, q_{i}\right) \mid \omega^{\prime}\right)\left[v_{q}\left(\omega^{\prime}, q_{i}\right)-\phi\left(p\left(\omega^{\prime}, q_{i}\right), \omega\right)\right],
\end{aligned}
$$

where the inequality follows from $s_{i}^{\prime}\left(\omega^{\prime}\right)<0$ from Lemma 1 and the reverse hazard rate dominance, $\phi\left(p\left(\omega^{\prime}, q_{i}\right), \omega^{\prime}\right)<\phi\left(p\left(\omega^{\prime}, q_{i}\right), \omega\right)$. Then this implies $v_{q}\left(\omega^{\prime}, q_{i}\right)-\phi\left(p\left(\omega^{\prime}, q_{i}\right), \omega\right)<0$. Hence, we have

$$
u_{\omega^{\prime}}\left(\omega^{\prime}, \omega, q_{i}\right)=-s_{i}^{\prime}\left(\omega^{\prime}\right) f\left(p\left(\omega^{\prime}, q_{i}\right) \mid \omega\right)\left[v_{q}\left(\omega^{\prime}, q_{i}\right)-\phi\left(p\left(\omega^{\prime}, q_{i}\right), \omega\right)\right]<0 .
$$

Similarly, one can show that for any $\omega^{\prime}<\omega, u_{\omega^{\prime}}\left(\omega^{\prime}, \omega, q_{i}\right)>0$. Together, the global sufficiency for the manager's truthful report is satisfied. Now, from (40), we find $s_{i}(\omega)$ explicitly such that

$$
\frac{s_{i}^{\prime}(\omega)}{s_{i}(\omega)}=\frac{p_{\omega}\left(\omega, q_{i}\right)}{p\left(\omega, q_{i}\right)-v_{q}\left(\omega, q_{i}\right)}, \text { or } \frac{\partial\left[\ln s_{i}(\omega)\right]}{\partial \omega}=\frac{p_{\omega}\left(\omega, q_{i}\right)}{p\left(\omega, q_{i}\right)-v_{q}\left(\omega, q_{i}\right)} .
$$

By taking the integral of both sides, we have

$$
\int_{\omega}^{\bar{\omega}} \frac{\partial\left[\ln s_{i}(x)\right]}{\partial x} d x=\int_{\omega}^{\bar{\omega}} \frac{p_{\omega}\left(x, q_{i}\right)}{p\left(x, q_{i}\right)-v_{q}\left(x, q_{i}\right)} d x
$$

which yields (24):

$$
\ln s_{i}(\omega)=\ln \bar{s}_{i}-\int_{\omega}^{\bar{\omega}} \frac{p_{\omega}\left(x, q_{i}\right)}{p\left(x, q_{i}\right)-v_{q}\left(x, q_{i}\right)} d x, \text { so } s_{i}(\omega)=\bar{s}_{i} \exp \left\{\int_{\omega}^{\bar{\omega}} \frac{p_{\omega}\left(x, q_{i}\right)}{v_{q}\left(x, q_{i}\right)-p\left(x, q_{i}\right)} d x\right\} .
$$

For the uniqueness, the partial derivative of $u\left(\omega^{\prime}, \omega, q_{i}\right)$ with respect to $\omega^{\prime}$ yields

$$
u_{\omega^{\prime}}\left(\omega^{\prime}, \omega, q_{i}\right)=-s_{i}^{\prime}\left(\omega^{\prime}\right) f\left(p\left(\omega^{\prime}, q_{i}\right) \mid \omega\right)\left[-\frac{s_{i}\left(\omega^{\prime}\right) p_{\omega}\left(\omega^{\prime}, q_{i}\right)}{s_{i}^{\prime}\left(\omega^{\prime}\right)}-\frac{F\left(p\left(\omega^{\prime}, q_{i}\right) \mid \omega\right)}{f\left(p\left(\omega^{\prime}, q_{i}\right) \mid \omega\right)}\right] .
$$

For any $s_{i}(\omega)$ satisfying the FOC, by the second best solution in (8),

$$
-\frac{s_{i}(\omega)}{s_{i}^{\prime}(\omega)}=\frac{F\left(p\left(\omega, q_{i}\right) \mid \omega\right)}{p_{\omega}\left(\omega, q_{i}\right) f\left(p\left(\omega, q_{i}\right) \mid \omega\right)}=\frac{v_{q}\left(\omega, q_{i}\right)-p\left(\omega, q_{i}\right)}{p_{\omega}\left(\omega, q_{i}\right)}
$$

which is identical to the first-order alignment in (24).

Part 2. Dominance. By choosing $\underline{S}=B+c$, we can choose $\left(\bar{s}_{0}, \ldots, \bar{s}_{N-1}\right)$ such that IR binds:

$$
B+c+\int_{\Omega} \sum_{i=0}^{N-1} \bar{s}_{i} \exp \left\{\int_{\omega}^{\bar{\omega}} \frac{p_{\omega}\left(x, q_{i}\right)}{v_{q}\left(x, q_{i}\right)-p\left(x, q_{i}\right)} d x\right\} F\left(p\left(\omega, q_{i}\right) \mid \omega\right) d G(\omega)=\bar{U}+c,
$$

where $\bar{U}-B>0$. 
Proof of Theorem 2. Part 1. First, it is clear that $\underline{S}=B+c$. Furthermore, by denoting $x_{i} \equiv \frac{\bar{s}_{i} U\left(q_{i}\right)}{\bar{U}-B+R}$ for all $i$ and $x=\left(x_{0}, \ldots, x_{\bar{N}-1}\right)$ with $\sum_{i=0}^{\bar{N}-1} x_{i}=1$, the maximization problem in (28) can be succinctly rewritten as

$$
\underset{x \in \Delta}{\operatorname{minimize}} \max _{\widehat{\omega} \in \Omega} \sum_{i=0}^{\bar{N}-1} x_{i} \frac{\widehat{U}\left(\widehat{\omega}, q_{i}\right)}{U\left(q_{i}\right)},
$$

given a compact set $\Delta \equiv\left\{x \in \mathbb{R}_{+}^{\bar{N}}: \sum_{i=0}^{\bar{N}-1} x_{i}=1\right\}$. For each $x$, by the Weierstrass theorem, there exists a solution, not necessarily a unique one. Still, by the maximum theorem, the objective function $\max _{\widehat{\omega} \in \Omega} \sum_{i=0}^{\bar{N}-1} x_{i} \frac{\widehat{U}\left(\widehat{\omega}, q_{i}\right)}{U\left(q_{i}\right)}$ is a continuous function of $x$. Since $x$ is from a compact set, there exists a solution to the minimax problem in (41).

Part 2. For a solution $x^{*}$ to the reformulation in (41), there is a unique $s^{*} \equiv\left(\bar{s}_{0}^{*}, \ldots, \bar{s}_{\bar{N}-1}^{*}\right)$ through $x_{i} \equiv \frac{\bar{s}_{i} U\left(q_{i}\right)}{\bar{U}-B+R}$, and vice versa. By the binding IR from (25) and (26),

$$
\begin{aligned}
B+c+\sum_{i=0}^{N-1} \bar{s}_{i}^{*} \widehat{U}\left(\widehat{\omega}, q_{i}\right) & =B+c+(\bar{U}-B+R)\left[\sum_{i=0}^{N-1} \frac{\bar{s}_{i}^{*}}{\bar{U}-B+R} \widehat{U}\left(\widehat{\omega}, q_{i}\right)\right] \\
& =B+c+(\bar{U}-B+R)\left[\sum_{i=0}^{N-1} x_{i}^{*} \frac{\widehat{U}\left(\widehat{\omega}, q_{i}\right)}{U\left(q_{i}\right)}\right],
\end{aligned}
$$

where the last line yields the formula as a linear function of $R$, since for each $i, x_{i}^{*}=\frac{\bar{s}_{i}^{*} U\left(q_{i}\right)}{\bar{U}-B+R}$.

Proof of Lemma 2. Part 1. By the binding IR from (25) and (26),

$$
\begin{aligned}
k(R) & =\bar{U}+R+c-\left[B+c+\sum_{i=0}^{N-1} \bar{s}_{i}^{*} \widehat{U}\left(\widehat{\omega}, q_{i}\right)\right] \\
& =(\bar{U}-B+R)\left[1-\sum_{i=0}^{N-1} x_{i}^{*} \frac{\widehat{U}\left(\widehat{\omega}, q_{i}\right)}{U\left(q_{i}\right)}\right],
\end{aligned}
$$

where the first equality follows from the binding IR $\int_{\Omega} \mathbb{E}_{\theta}[S(\omega, \theta) \mid \omega] d G(\omega)=\bar{U}+R+c$ and the second from Theorem 2. Then, $k(R)$ is continuous and strictly increasing.

Part 2. (i) The simultaneous contracting versus the ex ante contracting. If $c<k(\Delta U)$, for $0 \leq R<\Delta U$, we have $R<\Delta U \Leftrightarrow V_{s}-\bar{U}-R-c>V_{\min }-B-c$.

(ii) The simultaneous contracting versus the partial contracting. If $c<k\left(V_{s}-\bar{U}-c-V_{p}\right)$, for $0 \leq R<V_{s}-\bar{U}-c-V_{p}$, we have $R<V_{s}-\bar{U}-c-V_{p} \Leftrightarrow V_{s}-\bar{U}-R-c>V_{p}$. Since $c$ is strictly increasing, whereas $k\left(V_{s}-\bar{U}-c-V_{p}\right)$ is strictly decreasing in $c$, there is a unique fixed point $\widehat{c}$ satisfying $\widehat{c}=k\left(V_{s}-\bar{U}-V_{p}-\widehat{c}\right)$ such that for each $c<\widehat{c}, c<k\left(V_{s}-\bar{U}-c-V_{p}\right)$.

Now, $k(0)<c<k(\widehat{R})$, the optimal rent for the principal is the minimum $R$ such that $c=k(R)$. If $c \leq k(0)$, on the other hand, it is $R=0$.

Proof of Theorem 3. For the proof, we denote by $\succ_{D}$ the organizational dominance, together with notations for the three mechanisms such as SC for the simultaneous contracting; EC for the ex ante contracting; and $\mathrm{PC}$ for the partial contracting. First, note that $k(\Delta U)<V_{s}-\bar{U}-V_{p}$ is satisfied if $\bar{U}-B+\Delta U$ is sufficiently small. Then by Lemma 2 ,

$$
\begin{cases}S C \succ_{D} E C & \text { if } c<k(\Delta U), \\ E C \succ_{D} S C & \text { if } c>k(\Delta U),\end{cases}
$$


and, by comparing $E C$ with $P C$, it is straightforward to find a cut-off $V_{\min }-B-V_{p}$ such that

$$
\begin{cases}E C \succ_{D} P C & \text { if } c<V_{\min }-B-V_{p} \\ P C \succ_{D} E C & \text { if } c>V_{\min }-B-V_{p}\end{cases}
$$

Step 1. Show the optimal mechanism. We show it first for (i) of the Theorem given three mechanisms, and next for (ii) given two mechanisms.

Case 1. $k(\Delta U)<V_{\min }-B-V_{p}$. Then for $c<k(\Delta U), S C \succ_{D} E C$ and $E C \succ_{D} P C$, so $S C \succ_{D} E C \succ_{D} P C$; for $k(\Delta U)<c<V_{\min }-B-V_{p}, E C \succ_{D} S C$ and $E C \succ_{D} P C$; and for $c>V_{\min }-B-V_{p}, E C \succ_{D} S C$ and $P C \succ_{D} E C$, so $P C \succ_{D} E C>_{D} S C$. This yields the first set of the optimal organization structure.

Case 2. $k(\Delta U)>V_{\min }-B-V_{p}$. Then for $c<V_{\min }-B-V_{p}, S C \succ_{D} E C$ and $E C \succ_{D} P C$, so $S C \succ_{D} E C \succ_{D} P C$; and for $c>k(\Delta U), E C \succ_{D} S C$ and $P C \succ_{D} E C$, so $P C \succ_{D}>E C \succ_{D} S C$. Since for $V_{\min }-B-V_{p}<c<k(\Delta U), S C \succ_{D} E C$ and $P C \succ_{D} E C$, it remains to determine whether $S C$ dominates $P C$ or not. First, show that $\widehat{c} \in\left(V_{\min }-B-V_{p}, k(\Delta U)\right)$. Recall that by Lemma $2, \widehat{c}=k\left(V_{s}-\bar{U}-V_{p}-\widehat{c}\right)$. If $c=k(\Delta U)$, then

$$
k\left(V_{s}-\bar{U}-V_{p}-k(\Delta U)\right)=k\left(\Delta U+V_{\min }-B-V_{p}-k(\Delta U)\right)<k(\Delta U)=c,
$$

which implies that $\widehat{c}<k(\Delta U)$ since $c-k\left(V_{s}-\bar{U}-V_{p}-c\right)$ is a strictly increasing function of $c$. Similarly, if If $c=V_{\min }-B-V_{p}$, then

$$
k\left(V_{s}-\bar{U}-V_{p}-\left(V_{\min }-B-V_{p}\right)\right)=k(\Delta U)>V_{\min }-B-V_{p}=c,
$$

which implies that $\widehat{c}>V_{\min }-B-V_{p}$. Then it follows from Lemma 2 that for $c<\widehat{c}, S C \succ_{D} P C$, whereas for $c>\widehat{c}, P C \succ_{D} S C$.

Step 2. Show that the first case (i) exists for some $B$. For a sufficiently small negative $B$,

$$
k(\Delta U)=\left(V_{s}-V_{\min }\right)\left[1-\underset{x \in \Delta}{\operatorname{minimize}} \max _{\widehat{\omega} \in \Omega} \sum_{i=0}^{\bar{N}-1} x_{i} \frac{\widehat{U}\left(\widehat{\omega}, q_{i}\right)}{U\left(q_{i}\right)}\right]<V_{\min }-B-V_{p}
$$

and it follows from $\Delta U>0$ that $V_{\min }-B-V_{p}<V_{s}-\bar{U}-V_{p}$.

\section{References}

Arrow, K.J. (1972), Control in Large Organizations, Management Science, 10, 397-408.

Alchian, A.A. and Demsetz, H. (1972), Production, Information Costs, and Economic Organization, American Economic Review, 62, 777-795.

Baker, G., Gibbons, R. and Murphy, K.J. (1994), Subjective Performance Measures in Optimal Incentive Contracts, Quarterly Journal of Economics, 109, 1125-1156.

Baron, D. and Myerson, R. (1982), Regulating a Monopolist with Unknown Costs, Econometrica, 50, 911930. 
Ben-Porath, E., Dekel, E and Lipman, B.L. (2014), Optimal Allocation With Costly Verification, American Economic Review, 104, 3779-3813.

Bergemann, D. and Välimäki, J. (2002), Information Acquisition and Efficient Mechanism Design, Econometrica, 70, 1007-1033.

Bénabou, R and Tirole, J. (2016), Bonus Culture: Competitive Pay, Screening, and Multitasking, Journal of Political Economy, 124, 305-370.

Burks, S., Cowgill, B., Hoffman, M. and Housman, M. (2015), The Value of Hiring through Referrals, Quarterly Journal of Economics, 130, 805-839.

Caplin, A. and Dean, M. (2015), Revealed Preference, Rational Inattention, and Costly Information Acquisition, American Economic Review, 105, 2183-2203.

Clark, K.B., Chew, W.B. and Fujimoto, T. (1987), Product Development in the World Auto Industry, Brookings Papers on Economic Activity, 729-781.

Crawford, V. and Sobel, J. (1982), Strategic Information Transmission, Econometrica, 50, 1431-1451.

Crémer, J., Khalil. F and Rochet, J.-C. (1998a), Contracts and Productive Information Gathering, Games and Economic Behavior, 25, 174-193.

Crémer, J., Khalil. F and Rochet, J.-C. (1998b), Strategic Information Gathering Before a Contract is Offered, Journal of Economic Theory, 81, 163-200.

Crémer, J. and McLean, R. P. (1988), Full Extraction of the Surplus in Bayesian and Dominant Strategy Auctions, Econometrica, 56, 1247-1257.

Deb, J., Li, J. and Mukherjee, A. (2016), Relational Contracts with Subjective Peer Evaluations, RAND Journal of Economics, 47, 3-28.

Fuchs, W. (2007), Contracting with Repeated Moral hazard and Private Evaluations, American Economic Review, 97, 1432-1448.

Grossman, S. and Hart, O. (1986), The Costs and Benefits of Ownership: A Theory of Vertical and Lateral Integration, Journal of Political Economy, 94, 691-719.

Hart, O. and Moore, J. (1990), Property Rights and the Nature of the Firm, Journal of Political Economy, 98, 1119-1158.

Holmström, B. (1977), On Incentives and Control in Organizations (Ph.D. Thesis, Stanford University).

Holmström, B. (1984), On the Theory of Delegation, in M. Boyer and R. Kihlstrom (eds.) Bayesian Models in Economic Theory (New York: North-Holland), 115-141.

Holmström, B. and Milgrom, P. (1991), Multitask Principal-Agent Analyses: Incentive Contracts, Asset Ownership, and Job Design, Journal of Law, Economics, and Organization, 7, 24-52.

Holmström, B. and Roberts, J. (1999), Boundaries of the Firm Revisited, Journal of Economic Perspectives, $12,73-94$. 
Innes, R. D. (1990), Limited Liability and Incentive Contracting with Ex-Ante Action Choices, Journal of Economic Theory, 52, 45-67.

Kirkegaard, R. (2012), A Mechanism Design Approach to Ranking Asymmetric Auctions , Econometrica, 80, 2349-2364.

Kreps, D. (1990), Corporate culture and economic theory. In: J. Alt and K. Shepsle (eds.): Perspectives on Positive Political Economy, Cambridge, U.K.: Cambridge University Press.

Krishna, V. (2002) Auction Theory (Academic Press).

Levin, J. (2003), Relational Incentive Contracts, American Economic Review, 93, 835-857.

MacLeod, W. B. (2003), Optimal Contracting with Subjective Evaluation, American Economic Review, 93, $216-240$.

Maskin, E. and Riley, J. (2000), Asymmetric Auctions, Review of Economic Studies, 67, 413-438.

Mussa, M. and Rosen, S. (1978), Monopoly and Product Quality, Journal of Economic Theory, 18, 301-317.

Myerson, R. (1982), Optimal Coordination Mechanisms in Generalized Principal-Agent Problems, Journal of Mathematical Economics, 10, 67-81.

Nickell, S. (1997), Unemployment and Labor Market Rigidities: Europe versus North America, Journal of Economic Perspectives, 11, 55-74.

Poblete, J., and D. Spulber (2012), The Form of Incentive Contracts: Agency With Moral Hazard, Risk Neutrality, and Limited Liability, The RAND Journal of Economics, 43, 215-234.

Pallais, A. and Sands, E. (2016), Why the Referential Treatment? Evidence from Field Experiments on Referrals, Journal of Political Economy, 124, 1793-1828.

Rahman, D. (2012), But Who Will Monitor the Monitor?, American Economic Review, 102, 2767-2797

Rebick, M.E. (1993), The Persistence of Firm-Size Earnings Differentials and Labor Market Segmentation in Japan, Journal of the Japanese and International Economies, 7, 132-156.

Schmitz, P.W. (2002), On the Interplay of Hidden Action and Hidden Information in Simple Bilateral Trading Problems, Journal of Economic Theory, 103, 444-460.

Strausz, R. (2012), Mediated Contracts and Mechanism Design, Journal of Economic Theory, 147, 12801290.

Tirole, J. (1986), Hierarchies and Bureaucracies: On the Role of Collusion in Organization. Journal of Law, Economics, and Organization, 2, 181-214.

Williamson, O. (1975), Markets and Hierarchies: Analysis of Antitrust Implications (New York: Free Press). Williamson, O. (1985), The Economic Institutions of Capitalism (New York: Free Press).

Yoo, S.H. (2017), Optimal Design for an Informed Auctioneer, a working paper. 


\section{Appendix B For Online Publication Only}

\section{B.1 Mechanisms that make both $\mathrm{M}$ and A report $\omega$}

If both the manager and the agent report $\omega$, a direct mechanism consists of functions $\widehat{q}, \widehat{t}$ and $\widehat{S}$ where $\widehat{q}: \Theta \times \Omega^{2} \rightarrow \mathbb{R}_{+}, \widehat{t}: \Theta \times \Omega^{2} \rightarrow \mathbb{R}$ and $\widehat{S}: \Omega \times \Theta \times \Omega \rightarrow \mathbb{R}$. If the agent reports $\left(\theta, \omega^{\prime \prime}\right)$, and the manager reports $\omega^{\prime}$, then the principal assigns the agent the production of $\widehat{q}\left(\theta, \omega^{\prime \prime}, \omega^{\prime}\right)$ and commits to paying $\widehat{t}\left(\theta, \omega^{\prime \prime}, \omega^{\prime}\right)$ to the agent and $\widehat{S}\left(\omega^{\prime}, \theta, \omega^{\prime \prime}\right)$ to the manager.

Proposition 4 If the manager's contract in a mechanism does not depend on $\theta$, for any true $\omega$, any report $\widehat{\omega}$ can be an equilibrium in the mechanism. If the manager's contract in a mechanism depends on $\theta$, to induce a unique Bayesian equilibrium, the principal elicits reporting on the quality only from the manager.

Proof. Case 1. Suppose the manager's contract does not depend on $\theta$. If it is incentive compatible, then for each truthful report of $\theta \in \Theta$, the following conditions are satisfied: for each true $\omega \in \Omega$ and every reports $\omega^{\prime}, \omega^{\prime \prime} \in \Omega, \widehat{S}(\omega, \theta, \omega) \geq \widehat{S}\left(\omega^{\prime}, \theta, \omega\right)$ for the manager, and $\widehat{t}(\theta, \omega, \omega)-\widehat{q}(\theta, \omega, \omega) \theta \geq$ $\widehat{t}\left(\theta, \omega^{\prime \prime}, \omega\right)-\widehat{q}\left(\theta, \omega^{\prime \prime}, \omega\right) \theta$ for the agent. However, the same incentive compatibility allows all other $\widehat{\omega} \neq \omega$ to arise as an equilibrium because the true quality affects neither directly: that is, for each reports $\omega^{\prime}, \omega^{\prime \prime} \in \Omega, \widehat{S}(\widehat{\omega}, \theta, \widehat{\omega}) \geq \widehat{S}\left(\omega^{\prime}, \theta, \widehat{\omega}\right)$ and $\widehat{t}(\theta, \widehat{\omega}, \widehat{\omega})-\widehat{q}(\theta, \widehat{\omega}, \widehat{\omega}) \theta \geq \widehat{t}\left(\theta, \omega^{\prime \prime}, \widehat{\omega}\right)-\widehat{q}\left(\theta, \omega^{\prime \prime}, \widehat{\omega}\right) \theta$.

Case 2. Now, suppose the manager's contract depends on $\theta$. If a direct mechanism $(\widehat{q}, \widehat{t}, \widehat{S})$ is incentive compatible, then for each truthful report of $\theta \in \Theta$, the following conditions are satisfied for any $\omega \neq \omega^{\prime} \in \Omega$ : (i) for the manager,

$$
\mathbb{E}_{\theta}[\widehat{S}(\omega, \theta, \omega) \mid \omega] \geq \mathbb{E}_{\theta}\left[\widehat{S}\left(\omega^{\prime}, \theta, \omega\right) \mid \omega\right]
$$

and (ii) for the agent, it is identical to the one from Case 1. It is sufficient to show that eliciting the quality from both the manager and the agent leads to a continuum of non-truthful equilibria. The manager's truthful report requires an additional incentive compatibility condition: for each $\omega \in \Omega$, there exists $\epsilon>0$ such that for any $\omega^{\prime} \in(\omega-\epsilon, \omega+\epsilon)$,

$$
\mathbb{E}_{\theta}\left[\widehat{S}\left(\omega^{\prime \prime}, \theta, \omega^{\prime}\right) \mid \omega\right] \leq \mathbb{E}_{\theta}\left[\widehat{S}\left(\omega^{\prime}, \theta, \omega^{\prime}\right) \mid \omega\right] \text { for all } \omega^{\prime \prime} \in \Omega \text { and } \omega^{\prime \prime} \neq \omega^{\prime} .
$$

If not, there exists $\omega \in \Omega$ such that for each $\epsilon>0$, there exists $\omega^{\prime} \in(\omega-\epsilon, \omega+\epsilon)$ such that

$$
\mathbb{E}_{\theta}\left[\widehat{S}\left(\omega^{\prime \prime}, \theta, \omega^{\prime}\right) \mid \omega\right]>\mathbb{E}_{\theta}\left[\widehat{S}\left(\omega^{\prime}, \theta, \omega^{\prime}\right) \mid \omega\right] \text { for some } \omega^{\prime \prime} \in \Omega \text { and } \omega^{\prime \prime} \neq \omega^{\prime} .
$$

This implies:

$$
\mathbb{E}_{\theta}\left[\widehat{S}\left(\omega^{\prime \prime}, \theta, \omega^{\prime}\right) \mid \omega^{\prime}\right]>\mathbb{E}_{\theta}\left[\widehat{S}\left(\omega^{\prime}, \theta, \omega^{\prime}\right) \mid \omega^{\prime}\right] \text { for some } \omega^{\prime \prime} \in \Omega \text { and } \omega^{\prime \prime} \neq \omega^{\prime},
$$

which is a contradiction with the condition for the manager's truth-telling of $\omega^{\prime}$ from (42). Hence, (43) implies that for each $\omega \in \Omega$, there is a continuum of non-truthful equilibria, both reporting $\left(\omega^{\prime}, \omega^{\prime}\right)$ for $\omega^{\prime} \neq \omega$.

For each true $\omega \in \Omega$, any $\widehat{\omega} \in \Omega$ arises as an equilibrium if the manager's contract in a mechanism does not depend on $\theta$, because true quality $\omega$ is not embedded in either the manger's payoff or the agent's. Hence, with a positive information cost, if the manager chooses $\widehat{\omega}$ maximizing $\widehat{S}(\omega, \theta, \omega)$ since any $\widehat{\omega} \in \Omega$ arises as an equilibrium, then

$$
\widehat{S}(\widehat{\omega}, \theta, \widehat{\omega}) \geq \widehat{S}(\omega, \theta, \omega)>\widehat{S}(\omega, \theta, \omega)-c,
$$

which fails to incentivize the manager's information acquisition. 


\section{B.2 Omitted steps between (20) and (21)}

For $(16) \Rightarrow(17)$, we provide omitted steps between $(20)$ and $(21)$. We factor out $p_{\omega}\left(\omega^{\prime}, q_{i}\right)$ from (20) to have: for $\omega^{\prime}=\omega \in \Omega$,

$$
p_{\omega}\left(\omega^{\prime}, q_{i}\right)\left[\frac{v_{q}\left(\omega^{\prime}, q\right)-p\left(\omega^{\prime}, q_{i}\right)}{p_{\omega}\left(\omega^{\prime}, q_{i}\right)}-\frac{F\left(p\left(\omega^{\prime}, q_{i}\right) \mid \omega\right)}{f\left(p\left(\omega^{\prime}, q_{i}\right) \mid \omega\right) p_{\omega}\left(\omega^{\prime}, q_{i}\right)}\right]=0,
$$

which can be rewritten as

$$
p_{\omega}\left(\omega^{\prime}, q_{i}\right)\left[\frac{v_{q}\left(\omega^{\prime}, q_{i}\right)-p\left(\omega^{\prime}, q_{i}\right)}{p_{\omega}\left(\omega^{\prime}, q_{i}\right)}-\frac{F\left(p\left(\omega^{\prime}, q_{i}\right) \mid \omega\right)}{\partial\left[F\left(p\left(\omega^{\prime}, q_{i}\right) \mid \omega\right)\right] / \partial \omega^{\prime}}\right]=0,
$$

where note that to satisfy IC, $q=q_{s}\left(\theta, \omega^{\prime}\right)$ given $\theta>\underline{\theta}$ is necessary as discussed in (16). To satisfy the equality in (44), the term inside the above bracket must satisfy $\frac{p_{\omega}\left(\omega^{\prime}, q_{i}\right)}{v_{q}\left(\omega^{\prime}, q_{i}\right)-p\left(\omega^{\prime}, q_{i}\right)}-$ $\frac{\partial\left[F\left(p\left(\omega^{\prime}, q_{i}\right) \mid \omega\right)\right] / \partial \omega^{\prime}}{F\left(p\left(\omega^{\prime}, q_{i}\right) \mid \omega\right)}=0$, by switching the numerator with the denominator, which can be further rewritten as $\frac{p_{\omega}\left(\omega^{\prime}, q_{i}\right)}{v_{q}\left(\omega^{\prime}, q_{i}\right)-p\left(\omega^{\prime}, q_{i}\right)}-\frac{\partial \log \left(F\left(p\left(\omega^{\prime}, q_{i}\right) \mid \omega\right)\right)}{\partial \omega^{\prime}}=0$. Then, by taking the integral of the both sides, this implies

$$
\frac{\partial\left[\int_{\omega^{\prime}}^{\bar{\omega}} \frac{p_{\omega}\left(x, q_{i}\right)}{v_{q}\left(x, q_{i}\right)-p\left(x, q_{i}\right)} d x+\log \left(F\left(p\left(\omega^{\prime}, q_{i}\right) \mid \omega\right)\right)\right]}{\partial \omega^{\prime}}=0 \text { if } \omega^{\prime}=\omega \in \Omega,
$$

which, by having $\log \left(\exp \left\{\int_{\omega^{\prime}}^{\bar{\omega}} \frac{p_{\omega}\left(x, q_{i}\right)}{v_{q}\left(x, q_{i}\right)-p\left(x, q_{i}\right)} d x\right\}\right)$, leads to $(21)$.

The procedure to show IC for $q=q_{s}\left(\theta, \omega^{\prime}\right)$ given $\theta>\underline{\theta}$ is identical to the one in the proof of Theorem 1. We show that to satisfy IC, the integral must be taken from $\omega^{\prime}$ to $\bar{\omega}$ as in (22). Suppose, on the contrary, that we take the integral with the other direction to have

$$
\frac{\partial\left[\int_{\underline{\omega}}^{\omega^{\prime}} \frac{p_{\omega}\left(x, q_{i}\right)}{v_{q}\left(x, q_{i}\right)-p\left(x, q_{i}\right)} d x-\log \left(F\left(p\left(\omega^{\prime}, q_{i}\right) \mid \omega\right)\right)\right]}{\partial \omega^{\prime}}=0 \text { if } \omega^{\prime}=\omega \in \Omega .
$$

Note that given the opposite direction of the integral from $\underline{\omega}$ to $\omega^{\prime}$, we have minus sign in front of the second term in (46) unlike (45), and, to indicate the difference clearly, denote $\widehat{e}\left(\omega, q_{i}\right) \equiv$ $\exp \left\{\int_{\underline{\omega}}^{\omega} \frac{p_{\omega}\left(x, q_{i}\right)}{v_{q}\left(x, q_{i}\right)-p\left(x, q_{i}\right)} d x\right\}$ and $\widehat{u}\left(\omega^{\prime}, \omega, q_{i}\right)$ for the manager's expected payoff given $\widehat{e}\left(\omega, q_{i}\right)$, not $e\left(\omega, q_{i}\right)$ in $(22)$. The optimality condition in (46) is when the true quality is given as $\omega$, and the same optimality condition applies to the case that the true quality is $\omega^{\prime}$, which is given as

$$
\begin{aligned}
\widehat{u}_{\omega^{\prime}}\left(\omega^{\prime}, \omega^{\prime}, q_{i}\right)=0 & =\frac{p_{\omega}\left(\omega^{\prime}, q_{i}\right)}{v_{q}\left(\omega^{\prime}, q_{i}\right)-p\left(\omega^{\prime}, q_{i}\right)}-\frac{F\left(p\left(\omega^{\prime}, q_{i}\right) \mid \omega^{\prime}\right) p_{\omega}\left(\omega^{\prime}, q_{i}\right)}{f\left(p\left(\omega^{\prime}, q_{i}\right) \mid \omega^{\prime}\right)} \\
& <\frac{p_{\omega}\left(\omega^{\prime}, q_{i}\right)}{v_{q}\left(\omega^{\prime}, q_{i}\right)-p\left(\omega^{\prime}, q_{i}\right)}-\frac{F\left(p\left(\omega^{\prime}, q_{i}\right) \mid \omega\right) p_{\omega}\left(\omega^{\prime}, q_{i}\right)}{f\left(p\left(\omega^{\prime}, q_{i}\right) \mid \omega\right)},
\end{aligned}
$$

where the last inequality follows the reverse hazard rate dominance. This implies that for $\omega^{\prime}>\omega$,

$$
\widehat{u}_{\omega^{\prime}}\left(\omega^{\prime}, \omega, q_{i}\right)=\frac{\partial \widehat{e}\left(\omega^{\prime}, q_{i}\right)}{\partial \omega^{\prime}}-\frac{F\left(p\left(\omega^{\prime}, q_{i}\right) \mid \omega\right) p_{\omega}\left(\omega^{\prime}, q_{i}\right)}{f\left(p\left(\omega^{\prime}, q_{i}\right) \mid \omega\right)}>0
$$

and misreporting $\omega^{\prime}$ yields a higher payoff, violating IC. 


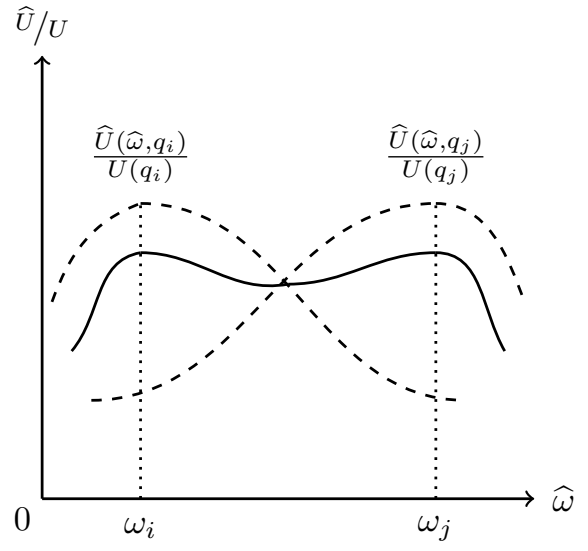

$(a)$

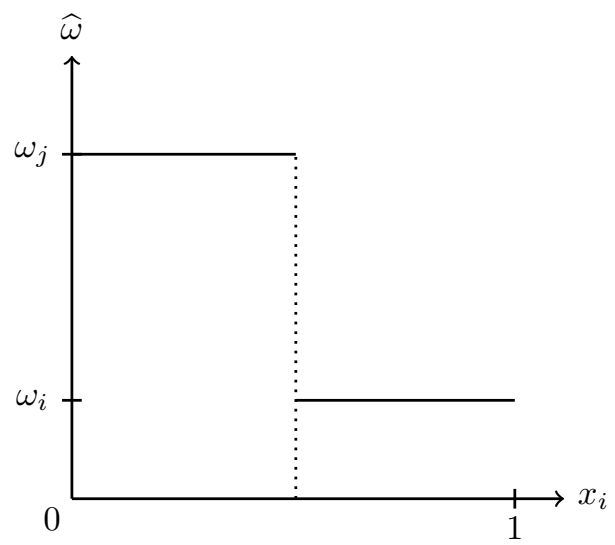

(b)

Figure 5: $x_{i} \frac{\widehat{U}\left(\widehat{\omega}, q_{i}\right)}{U\left(q_{i}\right)}+\left(1-x_{i}\right) \frac{\widehat{U}\left(\widehat{\omega}, q_{j}\right)}{U\left(q_{j}\right)}$ and not differentiable $\omega_{i j}^{*}\left(x_{i}\right)$

\section{B.3 Optimal number of intervals}

For its characterization, we suppose that each $\widehat{U}\left(\widehat{\omega}, q_{i}\right)$ has a single peak, which is denoted by $\omega_{i}$, that is, $\omega_{i}=\arg \max _{\widehat{\omega} \in \Omega} \widehat{U}\left(\widehat{\omega}, q_{i}\right)$. We say that an interval $q_{k}$ is dominant if for all $q_{j}$,

$$
\frac{\widehat{U}\left(\omega_{k}, q_{k}\right)}{U\left(q_{k}\right)} \leq \frac{\widehat{U}\left(\omega_{k}, q_{j}\right)}{U\left(q_{j}\right)}
$$

It is not difficult for one to envision a few figures to verify indeed that if there is a dominant interval, by the single-peak property, the single dominant interval solves the minimax problem.

A more general and interesting case is, however, that there is no such dominant interval, which invites a couple of challenges for the analysis: an arbitrary number of intervals and an irregularity of the function $\widehat{U}\left(\widehat{\omega}, q_{i}\right)$ with respect to $\widehat{\omega}$. If $\widehat{\omega}$ were fixed, that is, with no maximization problem in (41), the minimization problem itself is a simple linear programming with respect to $x=\left(x_{0}, \ldots, x_{\bar{N}-1}\right)$, like the cost minimization from classical consumer or production theory, thanks to the linear characterization of the solution $s_{i}(\omega)=\bar{s}_{i} e\left(\omega, q_{i}\right)$ from Theorem 1 . Despite the linearity, the minimax problem involves an additional layer of the optimization: for each vector of weights $x$, there is a corresponding $\widehat{\omega}$ that the manager chooses to deviate to maximize the convex combinations of the ratios, $\left(\frac{\widehat{U}\left(\widehat{\omega}, q_{i}\right)}{U\left(q_{i}\right)}\right)_{i=0, \ldots, \bar{N}-1}$. The maximized function $\max _{\widehat{\omega} \in \Omega} \sum_{i=0}^{\bar{N}-1} x_{i} \frac{\widehat{U}\left(\widehat{\omega}, q_{i}\right)}{U\left(q_{i}\right)}$ in the minimax problem is continuous in its parameter $x$ - so there exists a solution to the minimax problem - but if $\widehat{U}\left(\widehat{\omega}, q_{i}\right)$ is not concave for some $q_{i}$, its solution is not necessarily differentiable. ${ }^{42}$ On the other hand, conceivably, the concavity for all $i=0, \ldots, \bar{N}-1$ requires strong conditions on the distribution functions, $F$ and $G$, and $p$.

To delve into the challenges further, we probe the minimax problem only with two arbitrary intervals, as an intermediate step toward the main goal. The study on the two-interval case not only clarifies what the aforementioned difficulty means in detail but provides a clean segue into the second main result of this paper, the optimal number of intervals. The minimax with two intervals

\footnotetext{
${ }^{42}$ Technically, by the maximum theorem - the solution - the optimal $\widehat{\omega}$ is only upper hemicontinuous in $x$.
} 
$q_{i}$ and $q_{j}$ is given as

$$
\underset{x_{i} \in[0,1]}{\operatorname{minimize}} \max _{\widehat{\omega} \in \Omega} x_{i} \frac{\widehat{U}\left(\widehat{\omega}, q_{i}\right)}{U\left(q_{i}\right)}+\left(1-x_{i}\right) \frac{\widehat{U}\left(\widehat{\omega}, q_{j}\right)}{U\left(q_{j}\right)},
$$

where $\frac{\widehat{U}\left(\widehat{\omega}, q_{i}\right)}{U\left(q_{i}\right)}$ and $\frac{\widehat{U}\left(\widehat{\omega}, q_{j}\right)}{U\left(q_{j}\right)}$ have single peaks $\omega_{i}$ and $\omega_{j}$. We denote by $\omega_{i j}^{*}\left(x_{i}\right)$ a report $\widehat{\omega}$ satisfying the first-order condition for the manager's max problem to misreport worker quality $\widehat{\omega}$, given $x_{i}$ :

$$
x_{i} \frac{\widehat{U}_{\widehat{\omega}}\left(\omega_{i j}^{*}\left(x_{i}\right), q_{i}\right)}{U\left(q_{i}\right)}+\left(1-x_{i}\right) \frac{\widehat{U}_{\widehat{\omega}}\left(\omega_{i j}^{*}\left(x_{i}\right), q_{j}\right)}{U\left(q_{j}\right)}=0 .
$$

Provided that neither is a dominant interval, there is no problem (by the intermediate value theorem) of making $x_{i}$ generate an intersection between the two ratios as the one satisfying the equality above, but it may not satisfy the sufficiency; that is, the weight $x_{i}$ satisfying the first order optimality above in fact may not be a solution to the minimax. As an illustration, in Figure 4, consider a case that the convex combination of the two ratios is rather strictly convex between two peaks, $\omega_{i}$ and $\omega_{j}$. This particular case even exemplifies that the optimal $\widehat{\omega}$ is not a function, but a correspondence having multiple solutions, not differentiable, at least one $x_{i}$ value. Yet, the minimal condition to solve the minimax problem with the differentiability of $\widehat{\omega}$ with respect to $x_{i}$ is equivalent to the concavity on those between the two peaks.

Lemma 3 Suppose that $\widehat{U}\left(\widehat{\omega}, q_{i}\right)$ and $\widehat{U}\left(\widehat{\omega}, q_{j}\right)$ have single peaks with $\omega_{i}<\omega_{j}$ and that there is no dominant interval. Then, $\omega_{i j}^{*}\left(x_{i}\right)$ is an implicit function of $x_{i}$ on $[0,1]$ if and only if the convex combination of the two ratios is strictly concave on $\left[\omega_{i}, \omega_{j}\right]$ for $\omega_{i j}^{*}\left(x_{i}\right)$ satisfying the first-order condition.

Proof. For the proof, to simplify notations, denote the two ratios by

$$
\mathcal{R}_{i}(\widehat{\omega}) \equiv \frac{\widehat{U}\left(\widehat{\omega}, q_{i}\right)}{U\left(q_{i}\right)} \text { and } \mathcal{R}_{j}(\widehat{\omega}) \equiv \frac{\widehat{U}\left(\widehat{\omega}, q_{j}\right)}{U\left(q_{j}\right)} .
$$

First, show $(\Leftarrow)$. Suppose that for each $x_{i} \in[0,1], x_{i} \mathcal{R}_{i}(\widehat{\omega})+\left(1-x_{i}\right) \mathcal{R}_{j}(\widehat{\omega})$ is strictly concave on $\left[\omega_{i}, \omega_{j}\right]$ for $\omega_{i j}^{*}\left(x_{i}\right)$ satisfying the first-order condition. Then, such $\widehat{\omega}$ satisfies the second-order sufficiency. Furthermore, since for each $x_{i} \in[0,1], x_{i} \mathcal{R}_{i}^{\prime \prime}(\widehat{\omega})+\left(1-x_{i}\right) \mathcal{R}_{j}^{\prime \prime}(\widehat{\omega})<0$ for all $\widehat{\omega} \in\left[\omega_{i}, \omega_{j}\right]$, there exists a unique differentiable implicit function $\omega_{i j}^{*}\left(x_{i}\right)$ of $x_{i}$ on $[0,1]$. Show $(\Rightarrow)$. Suppose, on the contrary, that for some $x_{i}$, there exists one point $\omega^{\prime} \in\left[\omega_{i}, \omega_{j}\right]$ satisfying the first-order condition $x_{i} \mathcal{R}_{i}^{\prime}\left(\omega^{\prime}\right)+\left(1-x_{i}\right) \mathcal{R}_{j}^{\prime}\left(\omega^{\prime}\right)=0$ such that $x_{i} \mathcal{R}_{i}^{\prime \prime}\left(\omega^{\prime}\right)+\left(1-x_{i}\right) \mathcal{R}_{j}^{\prime \prime}\left(\omega^{\prime}\right) \geq 0$. If $x_{i} \mathcal{R}_{i}^{\prime \prime}\left(\omega^{\prime}\right)+\left(1-x_{i}\right) \mathcal{R}_{j}^{\prime \prime}\left(\omega^{\prime}\right)>$ 0 , then $\omega^{\prime}$ is not a solution. It remains to show it for $x_{i} \mathcal{R}_{i}^{\prime \prime}\left(\omega^{\prime}\right)+\left(1-x_{i}\right) \mathcal{R}_{j}^{\prime \prime}\left(\omega^{\prime}\right)=0$. The first-order condition for $\widehat{\omega}$ can be rewritten in the following way for $x_{i}$ such that

$$
x_{i}=\Phi_{i j}(\widehat{\omega}),
$$

where denote $\Phi_{i j}(\widehat{\omega}) \equiv-\frac{\mathcal{R}_{j}^{\prime}(\widehat{\omega})}{\mathcal{R}_{i}^{\prime}(\widehat{\omega})-\mathcal{R}_{j}^{\prime}(\widehat{\omega})}$. Hence, an existence of an implicit function is equivalent to an existence of an inverse function, which requires $\Phi_{i j}^{\prime}(\widehat{\omega}) \neq 0$. Note that

$$
\Phi_{i j}^{\prime}(\widehat{\omega})=-\frac{\mathcal{R}_{i}^{\prime}(\widehat{\omega}) \mathcal{R}_{j}^{\prime \prime}(\widehat{\omega})-\mathcal{R}_{j}^{\prime}(\widehat{\omega}) \mathcal{R}_{i}^{\prime \prime}(\widehat{\omega})}{\left[\mathcal{R}_{i}^{\prime}(\widehat{\omega})-\mathcal{R}_{j}^{\prime}(\widehat{\omega})\right]^{2}} .
$$


By substituting the first-order condition into $x_{i} \mathcal{R}_{i}^{\prime \prime}\left(\omega^{\prime}\right)+\left(1-x_{i}\right) \mathcal{R}_{j}^{\prime \prime}\left(\omega^{\prime}\right)=0$, we have $\mathcal{R}_{i}^{\prime}\left(\omega^{\prime}\right) \mathcal{R}_{j}^{\prime \prime}\left(\omega^{\prime}\right)-$ $\mathcal{R}_{j}^{\prime}\left(\omega^{\prime}\right) \mathcal{R}_{i}^{\prime \prime}\left(\omega^{\prime}\right)=0$ at $\omega^{\prime}$, which yields a contradiction.

The sufficiency for the implicit function is immediate; its necessity requires a formal proof, and more importantly, the consequence of Lemma 3 is that if $\omega_{i j}^{*}\left(x_{i}\right)$ is an implicit function of $x_{i}$ on $[0,1]$, then, a solution to the minimax problem in (48) with only two $q_{i}$ and $q_{j}$ must be an intersection of the two ratios. ${ }^{43}$ The intersection is denoted by $\omega_{i j}$ such that

$$
\frac{\widehat{U}\left(\omega_{i j}, q_{i}\right)}{U\left(q_{i}\right)}=\frac{\widehat{U}\left(\omega_{i j}, q_{j}\right)}{U\left(q_{j}\right)} .
$$

In other words, Lemma 3 enables us to obtain the natural outcome for the manager's optimal misreport: The solution to (48) arises at the intersection; for the optimal $x_{i}, \omega_{i j}^{*}\left(x_{i}\right)=\omega_{i j}$. There might be no such intersection between two peaks, but if there is, it must be unique given the single-peak property: for any $i, j, \frac{\widehat{U}\left(\widehat{\omega}, q_{i}\right)}{U\left(q_{i}\right)}-\frac{\widehat{U}\left(\widehat{\omega}, q_{j}\right)}{U\left(q_{j}\right)}$ is strictly decreasing on $\left(\omega_{i}, \omega_{j}\right)$.

Proposition 5 shows that if $\omega_{k l}^{*}\left(x_{k}\right)$ is an implicit function where targets $q_{k}$ and $q_{l}$ generate the minimum intersection, then the optimal number of intervals is just two; the two intervals solve the minimax problem in (41) with $\bar{N}$ potential intervals. Thus, the principal can use only two targets to solve the minimax, which enables the principal to economize a number of targets. Furthermore, the result minimizes the scope of the regularity for $\widehat{U}\left(\widehat{\omega}, q_{i}\right)$ : the strict concavity of the convex combination with the two ratios (between two peaks).

Proposition 5 Suppose that each $\widehat{U}\left(\widehat{\omega}, q_{i}\right)$ has a single peak and that there is no dominant interval. Then, if for $q_{k}, q_{l}$ solving $\min _{q_{i}, q_{j}} \frac{\widehat{U}\left(\omega_{i j}, q_{i}\right)}{U\left(q_{i}\right)}$, there exists an implicit function $\omega_{k l}^{*}\left(x_{k}\right)$ of $x_{k}$ on $[0,1]$, then two intervals given $q_{k}$ and $q_{l}$ solve the minimax problem.

Proof. Suppose, on the contrary, there is $x^{\prime} \in \Delta$ such that

$$
\max _{\widehat{\omega} \in \Omega} \sum_{i=0}^{\bar{N}-1} x_{i}^{\prime} \frac{\widehat{U}\left(\widehat{\omega}, q_{i}\right)}{U\left(q_{i}\right)}<\frac{\widehat{U}\left(\omega_{k l}, q_{k}\right)}{U\left(q_{k}\right)} .
$$

Since given $x^{\prime}$, the maximization yields $\max _{\widehat{\omega} \in \Omega} \sum_{i=0}^{\bar{N}-1} x_{i}^{\prime} \frac{\widehat{U}\left(\widehat{\omega}, q_{i}\right)}{U\left(q_{i}\right)} \geq \sum_{i=0}^{\bar{N}-1} x_{i}^{\prime} \frac{\widehat{U}\left(\omega_{k l}, q_{i}\right)}{U\left(q_{i}\right)}$, we have

$$
\frac{\widehat{U}\left(\omega_{k l}, q_{k}\right)}{U\left(q_{k}\right)}>\sum_{i=0}^{\bar{N}-1} x_{i}^{\prime} \frac{\widehat{U}\left(\omega_{k l}, q_{i}\right)}{U\left(q_{i}\right)} \geq \min \left\{\frac{\widehat{U}\left(\omega_{k l}, q_{0}\right)}{U\left(q_{0}\right)}, \ldots, \frac{\widehat{U}\left(\omega_{k l}, q_{\bar{N}-1}\right)}{U\left(q_{\bar{N}-1}\right)}\right\}
$$

Let the ratio from $q_{j}$ attain the min level, that is, $\frac{\widehat{U}\left(\omega_{k l}, q_{j}\right)}{U\left(q_{j}\right)}=\min \left\{\frac{\widehat{U}\left(\omega_{k l}, q_{0}\right)}{U\left(q_{0}\right)}, \ldots, \frac{\widehat{U}\left(\omega_{k l}, q_{\bar{N}-1}\right)}{U\left(q_{\bar{N}-1}\right)}\right\}$. Then, from the above, for $q_{j}$, we have

$$
\frac{\widehat{U}\left(\omega_{k l}, q_{j}\right)}{U\left(q_{j}\right)}<\frac{\widehat{U}\left(\omega_{k l}, q_{k}\right)}{U\left(q_{k}\right)}
$$

Note that the min function of $\widehat{\omega} \min \left\{\frac{\widehat{U}\left(\widehat{\omega}, q_{k}\right)}{U\left(q_{k}\right)}, \frac{\widehat{U}\left(\widehat{\omega}, q_{l}\right)}{U\left(q_{l}\right)}\right\}$ is related to the intersection of two areas under $\frac{\widehat{U}\left(\widehat{\omega}, q_{k}\right)}{U\left(q_{k}\right)}$ and $\frac{\widehat{U}\left(\widehat{\omega}, q_{l}\right)}{U\left(q_{l}\right)}$ in Figure 4. With the min function, the proof is divided into two cases.

\footnotetext{
${ }^{43}$ That is, the sufficiency for the implicit function follows from the implicit function theorem, and then, the intersection from the envelope theorem.
} 
Case 1. The single peak from $q_{j}, \omega_{j}$, is above the min function from $q_{k}$ and $q_{l}$ such that

$$
\frac{\widehat{U}\left(\omega_{j}, q_{j}\right)}{U\left(q_{j}\right)}>\min \left\{\frac{\widehat{U}\left(\omega_{j}, q_{k}\right)}{U\left(q_{k}\right)}, \frac{\widehat{U}\left(\omega_{j}, q_{l}\right)}{U\left(q_{l}\right)}\right\} .
$$

Consider two cases, $\omega_{j}>\omega_{k l}$ or $\omega_{j}<\omega_{k l}\left(\omega_{j} \neq \omega_{k l}\right.$ for $\widehat{U}\left(\widehat{\omega}, q_{j}\right)$ to be a function). Suppose $\omega_{j}>\omega_{k l}$. WLOG, let $\omega_{k}<\omega_{l}$. Then, by the above and (50),

$$
\frac{\widehat{U}\left(\omega_{k l}, q_{k}\right)}{U\left(q_{k}\right)}-\frac{\widehat{U}\left(\omega_{k l}, q_{j}\right)}{U\left(q_{j}\right)}>0 \text { and } \frac{\widehat{U}\left(\omega_{j}, q_{k}\right)}{U\left(q_{k}\right)}-\frac{\widehat{U}\left(\omega_{j}, q_{j}\right)}{U\left(q_{j}\right)}<0
$$

which, by the intermediate value theorem, implies that there is an intersection $\omega_{k j} \in\left(\omega_{k}, \omega_{j}\right)$ between two ratios $\frac{\widehat{U}\left(\widehat{\omega}, q_{k}\right)}{U\left(q_{k}\right)}$ and $\frac{\widehat{U}\left(\widehat{\omega}, q_{j}\right)}{U\left(q_{j}\right)}$. By the single-peak property, $\frac{\widehat{U}\left(\omega_{k j}, q_{k}\right)}{U\left(q_{k}\right)}<\frac{\widehat{U}\left(\omega_{k l}, q_{j}\right)}{U\left(q_{j}\right)}$. This contradicts that $\omega_{k l}$ generates the lowest intersection. The same argument applies to the case $\omega_{j}<\omega_{k l}$.

Case 2. The single peak from $q_{j}, \omega_{j}$, is below the min function from $q_{k}$ and $q_{l}$ such that

$$
\frac{\widehat{U}\left(\omega_{j}, q_{j}\right)}{U\left(q_{j}\right)} \leq \min \left\{\frac{\widehat{U}\left(\omega_{j}, q_{k}\right)}{U\left(q_{k}\right)}, \frac{\widehat{U}\left(\omega_{j}, q_{l}\right)}{U\left(q_{l}\right)}\right\} .
$$

We show that, in this case, for all $q_{i}, \frac{\widehat{U}\left(\omega_{j}, q_{i}\right)}{U\left(q_{i}\right)} \geq \frac{\widehat{U}\left(\omega_{j}, q_{j}\right)}{U\left(q_{j}\right)}$. Suppose not. That is, there is one $q_{h}$ such that for $\omega_{j}, \frac{\widehat{U}\left(\omega_{j}, q_{h}\right)}{U\left(q_{h}\right)}<\frac{\widehat{U}\left(\omega_{j}, q_{j}\right)}{U\left(q_{j}\right)}$. If there is no intersection not to have a contradiction as in Case 1 , it must be that for $\omega_{h}, \frac{\widehat{U}\left(\omega_{h}, q_{h}\right)}{U\left(q_{h}\right)} \leq \frac{\widehat{U}\left(\omega_{h}, q_{j}\right)}{U\left(q_{j}\right)}$. Then, we show that for all $q_{i}, \frac{\widehat{U}\left(\omega_{h}, q_{i}\right)}{U\left(q_{i}\right)} \geq \frac{\widehat{U}\left(\omega_{h}, q_{h}\right)}{U\left(q_{h}\right)}$. The same argument keeps applying until we reach the minimum single peak $q_{m}$ such that

$$
\frac{\widehat{U}\left(\omega_{i}, q_{i}\right)}{U\left(q_{i}\right)} \geq \frac{\widehat{U}\left(\omega_{m}, q_{m}\right)}{U\left(q_{m}\right)} \text { for all } i \in\{0, \ldots, \bar{N}-1\} .
$$

For the minimum single peak, there is no way to avoid an intersection except that for all $q_{i}$, $\frac{\widehat{U}\left(\omega_{m}, q_{i}\right)}{U\left(q_{i}\right)} \geq \frac{\widehat{U}\left(\omega_{m}, q_{m}\right)}{U\left(q_{m}\right)}$. But, in this case, the interval given $q_{m}$ is dominant, a contradiction.

The idea for the two-interval solution is in fact simple. If there exists an implicit function $\omega_{k l}^{*}\left(x_{k}\right)$ of $x_{k}$ on $[0,1]$, then for the two intervals, the manager chooses a unique intersection between the two ratios as the optimal report if he does not acquire the information. If the intersection is indeed the lowest intersection among all others, it solves the minimax problem; otherwise, the minimax point of a combination of some number - not necessarily two - of multiple ratios is lower than the minimax point of the two ratios, and thus, at least one single-peak function from the former must cut through one of the two ratios from the latter, contradicting that the latter yields the lowest minimax for any given two ratios.

To appreciate Proposition 5, we revisit Example 2, in which case in fact the sufficiency for the implicit function is satisfied for a uniform $G$; that is, each $\widehat{U}\left(\widehat{\omega}, q_{i}\right)$ is strictly concave on $\Omega .{ }^{44}$ This results in the following Corollary.

\footnotetext{
${ }^{44}$ The strict concavity can be found from the closed-form $\widehat{U}$, which is derived as, for a uniform $G$ with $[\underline{\omega}, \bar{\omega}]$,$$
\widehat{U}\left(\widehat{\omega}, q_{i}\right)=e^{(\bar{\omega}-\widehat{\omega})}\left(\frac{q_{i}-1}{q_{i}+1+\widehat{\omega}}\right)^{\left(q_{i}+1\right)}\left(\frac{\bar{\omega}+1}{\widehat{\omega}+1}\right) \frac{1}{\ln \left(\frac{\widehat{\omega}\left[\widehat{\omega}-\left(q_{i}+1\right)\right]}{(\hat{\omega}+1)\left(q_{i}+1\right)}\right)}\left[\left(\frac{\widehat{\omega}\left[\widehat{\omega}-\left(q_{i}+1\right)\right]}{(\widehat{\omega}+1)\left(q_{i}+1\right)}\right)^{\bar{\omega}}-\left(\frac{\widehat{\omega}\left[\widehat{\omega}-\left(q_{i}+1\right)\right]}{(\widehat{\omega}+1)\left(q_{i}+1\right)}\right)^{\underline{\omega}}\right] .
$$ 
Corollary 2 Consider Example 2 and a uniform $G$. Then, for any finite feasible intervals, the number of intervals to solve the optimal interval structure is at most two. 